CAE Working Paper \#04-17

Intergenerational Equity and the Forest Management Problem

by

Tapan Mitra

December 2004 


\title{
Intergenerational Equity and the Forest Management Problem.*
}

\author{
Tapan Mitra ${ }^{\dagger}$
}

\begin{abstract}
The paper re-examines the foundations of representation of intertemporal preferences that satisfy intergenerational equity, and provides an axiomatic characterization of those social welfare relations, which are representable by the utilitarian ordering, in ranking consumption sequences which are eventually identical. A maximal point of this ordering is characterized in a standard model of forest management. Maximal paths are shown to converge over time to the forest with the maximum sustained yield, thereby providing a theoretical basis for the tradition in forest management, which has emphasized the goal of maximum sustained yield. Further, it is seen that a maximal point coincides with the optimal point according to the well-known overtaking criterion. This result indicates that the more restrictive overtaking criterion is inessential for a study of forest management under intergenerational equity, and provides a more satisfactory basis for the standard forestry model.

Journal of Economic Literature Classification Numbers: C61, D60, D70, D90, O41, Q23.
\end{abstract}

*This paper owes much to my ongoing collaborative work with Kaushik Basu on the representation of intertemporal preferences, with Swapan Dasgupta on characterizing optimal policies in the forestry model, and with M. Ali Khan on the choice of technique in a dynamic economy. An earlier version of the paper was presented at the International Conference on the Economics of Sustainable Forest Management at the University of Toronto, May 20-22, 2004. The present version has benefited from detailed comments by Shashi Kant.

${ }^{\dagger}$ Department of Economics, Cornell University, Ithaca, NY 14853, Email: tm19@cornell.edu. 


\section{Introduction}

In forestry management, there has been a tradition which claims that "the goal of good policy is to have sustained forest yield, or even maximum sustained yield somehow defined" [Samuelson (1976, p.146)]. In an attempt to understand this tradition, Mitra and Wan (1986) formulated the problem of forest management as one of optimizing the sum of (undiscounted) utlities from harvests of timber according to the well-known overtaking criterion. Their formulation allowed forestry economics to be viewed as a particular case of modern capital theory. Exploiting the optimization methods familiar from this general theory, they were able to show that, when the utility function is strictly concave, starting from any initial forestry configuration, the optimally managed forest converges over time to the forest with the maximum sustained yield, which corresponds to the "golden-rule" of the forestry model. This demonstration provided a theoretical basis for the tradition in forestry management.

Over the last twenty five years, work on the general area of sustainable development has brought about a thorough re-examination of the nature of preferences over time relevant to studies on environmental economics and the management of renewable resources. The Pareto efficiency principle is, of course, an essential ingredient in such studies. And, the equal treatment of all generations figures prominently in this literature, unlike other optimal intertemporal allocation problems, where discounting future utilities is common practice. ${ }^{1}$

These two guiding principles in the study of intertemporal preferences (often jointly referred to as the Suppes-Sen grading principle $)^{2}$ do not, however, take us far in terms of being able to develop useful qualitative properties of the evolution of renewable resource stocks over time under appropriate management. In aggregating utilities of all generations, it is therefore common practice to choose a more restrictive social welfare relation (SWR), and typically this SWR has been induced by some form of the overtaking criterion.

In this paper, I will re-examine the foundations of intertemporal preferences which respect intergenerational equity, propose a social welfare relation

\footnotetext{
${ }^{1}$ Ramsey (1928) had maintained that discounting one generation's utility or income vis-a-vis another's to be "ethically indefensible", and something that "arises merely from the weakness of the imagination".

${ }^{2}$ The Grading Principle is due to Suppes (1966). For a comprehensive analysis of it, see Sen (1971).
} 
which is weaker (less restrictive) than the one induced by the overtaking criterion, and provide an axiomatic basis for it. I will then apply this SWR to a standard model of forestry and demonstrate the somewhat surprising result that all the qualitative properties of optimally managed forests that one can obtain by applying the more restrictive overtaking criterion can be obtained with the application of the weaker and more acceptable SWR.

In proposing the new SWR, I would like to push the point of view that in comparing infinite consumption streams, it is only the comparisons of consumption streams which are "eventually identical" that are truly noncontroversial. ${ }^{3}$ Consequently, I will put forward a set of axioms which are consistent with this point of view. These axioms are Weak Pareto, Anonymity, Completeness and Continuity for finite horizon comparisons, and Independence. Of these the first two have already been discussed above, except that we postulate a weak version of Pareto, which restricts comparisons to consumption streams which are eventually identical. The third axiom is natural: since we are, after all, extending the theory from the finite-dimensional to the infinite-dimensional case, we cannot hope to weaken the postulates of the standard finite-dimensional theory. ${ }^{4}$ In the intertemporal context, the fourth axiom is common in utilitarian representations of preferences.

A noteworthy feature of our SWR is that it is axiomatized without postulating any continuity property on the preference relation in the infinite dimensional space containing the set of consumption streams. ${ }^{5}$ In contrast, axiomatic characterizations of the more restrictive SWR induced by the overtaking criterion typically involve some form of a continuity axiom. ${ }^{6}$ Axioms on the continuity of preferences in infinite-dimensional spaces have been the most controversial in the literature in this area, since the topology in which such continuity is assumed determines to a large extent the nature of allowable preferences. ${ }^{7}$

\footnotetext{
${ }^{3}$ Such comparisons figure prominently in the theory of efficient capital accumulation of Malinvaud (1953), and in the theory of representation of intertemporal preferences of Koopmans (1972).

${ }^{4}$ For the standard finite dimensional theory, see Debreu (1959), which in turn is based on Debreu (1954).

${ }^{5}$ In this regard, the current work can be seen as a continuation of the study in Basu and Mitra (2003a).

${ }^{6}$ The study by Brock (1970b) uses a "consistency axiom" which is actually a continuity restriction on the underlying preferences. A more recent study by Asheim and Tungodden (2004) also uses a similar continuity axiom.

${ }^{7}$ This is in contrast to postulating continuity properties on preferences in the finite-
} 
We apply our SWR to rank consumption streams generated by the model of forestry used in Mitra and Wan(1986). We call a consumption stream maximal if it is a maximal point in the feasible set in terms of the SWR, and study properties of maximal paths. We find that maximal paths converge over time to the forest with the maximum sustained yield, which demonstrates that the notion of maximality is enough to provide a theoretical basis for the tradition in forest management, which has emphasized the goal of maximum sustained yield. That is, in studying the long-run properties of paths which are maximal and those which are optimal according to the familiar overtaking criterion, the latter concept does not possess any discernible advantage.

Using duality theory, we show that maximal paths have generalized intertemporal profit maximizing (bounded) shadow prices associated with them, just like optimal paths do. So, one cannot have "errant behavior" on maximal paths, compared to optimal paths, in the short-run either. We combine the above two findings to establish the result that the set of maximal paths coincides exactly with the set of optimal paths. This leads us to conclude that in the context of the forestry model, one can completely dispense with the more restrictive overtaking criterion.

\section{Notation}

Let $\mathbb{N}$ denote the set of natural numbers $\{1,2,3, \ldots\}$, and let $\mathbb{R}$ denote the set of real numbers. Let $L$ denote the closed interval $[0,1] \subset \mathbb{R}$, let $S$ denote the set $\mathbb{R}^{\mathbb{N}}$, and let $C$ denote the set $L^{\mathbb{N}}$. $C$ is to be interpreted as the set of consumption sequences. Thus, we write $c \equiv\left(c_{1}, c_{2}, \ldots\right) \in C$ if and only if $c_{t} \in[0,1]$ for all $t \in \mathbb{N}$.

Given $x \in S$, and $N \in \mathbb{N}$, let us denote by $x(N)$ the vector consisting of the first $N$ elements of $x$ and by $x[N]$ the sequence from term $(N+1)$ onwards. So, $x(N)=\left(x_{1}, x_{2}, \ldots, x_{N}\right)$ and $x[N]=\left(x_{N+1}, x_{N+2}, \ldots\right)$. Clearly, $x[N] \in S$. We will denote the sequence $\left(x_{1}, x_{2}, \ldots, x_{N}, 0,0, \ldots\right)$ by $(x(N), 0[N])$.

For $c, c^{\prime} \in C$, we write $c^{\prime} \geq c$ if $c_{t}^{\prime} \geq c_{t}$ for all $t \in \mathbb{N}$; and, we write $c^{\prime}>c$ if $c^{\prime} \geq c$, and $c^{\prime} \neq c$.

dimensional theory. 
For each $n \in \mathbb{N}$, the sum-norm on $\mathbb{R}^{n}$ is defined by:

$$
\|x\|=\sum_{i=1}^{n}\left|x_{i}\right| \text { for } x \in \mathbb{R}^{n}
$$

The corresponding sum metric is defined by:

$$
d(x, y)=\|x-y\| \text { for } x, y \in \mathbb{R}^{n}
$$

The unit vectors in $\mathbb{R}^{n}$ are denoted by $e^{1}, \ldots, e^{n}$.

Denote by $U$ the set of continuous functions $u: L \rightarrow \mathbb{R}$, such that $u$ is increasing on $L$, and $u(0)=0, u(1)=1$.

A social welfare relation (SWR) is a binary relation, $\succsim$, on $C$, which is reflexive and transitive (a pre-ordering). ${ }^{8}$ We associate with $\succsim$ its symmetric and asymmetric components in the usual way. Thus, we write $c \sim c^{\prime}$ when $c \succsim c^{\prime}$ and $c^{\prime} \succsim c$ both hold; and, we write $c^{\prime} \succ c$ when $c^{\prime} \succsim c$ holds, but $c \succsim c^{\prime}$ does not hold.

A SWR $\succsim_{A}$ is a subrelation to a SWR $\succsim_{B}$ if (a) $x, y \in X$ and $x \succsim_{A} y$ implies $x \succsim_{B} y$; and (b) $x, y \in X$ and $x \succ_{A} y$ implies $x \succ_{B} y$.

\section{On a Utilitarian Social Welfare Relation ${ }^{9}$}

In this section we will introduce the notion of a utilitarian social welfare relation, and provide an axiomatic basis for it. The social welfare relation will only compare consumption sequences which are "eventually identical", so that the relation will be "more incomplete" than the familiar SWR induced by the overtaking criterion. ${ }^{10}$ It will be based consequently on axioms that relate only to consumption sequences which are eventually identical, and therefore will be more widely acceptable.

\footnotetext{
${ }^{8}$ In the economics literature, a pre-ordering is often refereed to as a "partial ordering". However, in the mathematics literature, the term "partial ordering" refers to a binary relation which is transitive and antisymmetric. To avoid confusion, we use the mathematical terminology, since the term "pre-order" is never used in any other sense in either discipline. Incidentally, our usage coincides with the terminology introduced in Debreu (1959).

${ }^{9}$ The results in this section are stated without proofs. They can be established along the lines indicated in Mitra (2003).

${ }^{10}$ Our SWR will later be compared in detail with the one induced by the overtaking criterion.
} 


\subsection{Axiomatic Characterization of a Utilitarian SWR}

Definition 1 Given a utility function, $u \in U$, a utilitarian SWR corresponding to $u$ is a binary relation $\succsim_{u}$, defined by:

$$
\begin{gathered}
c \succsim_{u} c^{\prime} \text { iff } \exists N \in \mathbb{N} \text { such that } c[N]=c^{\prime}[N], \\
\text { and } \sum_{t=1}^{N} u\left(c_{t}\right) \geq \sum_{t=1}^{N} u\left(c_{t}^{\prime}\right)
\end{gathered}
$$

We find the ranking of consumption sequences according to the utilitarian SWR to be persuasive. Thus, one may consider getting together the members of the finite society $\{1, \ldots, N\}$, and asking them to rank $c$ versus $c^{\prime}$. If they apply utilitarian principles to themselves, they will rank $c$ above $c^{\prime}$ if:

$$
\sum_{t=1}^{N} u\left(c_{t}\right)>\sum_{t=1}^{N} u\left(c_{t}^{\prime}\right)
$$

In this case, it is legitimate for the infinite horizon society to rank $c$ above $c^{\prime}$ because the infinite number of future generations, who are not included in the finite society $\{1, \ldots, N\}$, are indifferent between $c$ and $c^{\prime}$. In other words, in this situation, all future generations beyond $N$ are willing to go along with the (utilitarian) preferences of the finite society $\{1, \ldots, N\}$.

The following example illustrates the nature of the utilitarian SWR:

\section{Example 1}

$$
\begin{aligned}
& c=\left(\begin{array}{lllllll}
0.2, & 0, & 0.1, & 0.2 & 0.1, & 0.2, & \ldots
\end{array}\right) \\
& c^{\prime}=\left(\begin{array}{lllllll}
0, & 0.1, & 0.1 & 0.2, & 0.1 & 0.2, & \ldots
\end{array}\right)
\end{aligned}
$$

Here the finite society $\{1,2\}$ applying utilitarian principles to itself prefers $c$ to $c^{\prime}$. All future generations beyond time period 2 are indifferent between $c$ and $c^{\prime}$. So, in this case, we would have $c \succ_{u} c^{\prime}$, according to every utility function $u \in U$.

We turn now to the set of axioms which characterize the utilitarian SWR. To this end, let us first consider the following two axioms, which are fairly straightforward.

Axiom 1 (Weak Pareto) If $c, c^{\prime} \in C$, and there exists $N \in \mathbb{N}$, such that $c(N)>c^{\prime}(N)$ and $c[N]=c^{\prime}[N]$, then $c \succ c^{\prime}$. 
Axiom 2 (Anonymity) If $c, c^{\prime}$ are in $C$, and there exist $r, s$ in $\mathbb{N}$, such that $c_{r}=c_{s}^{\prime}$ and $c_{s}=c_{r}^{\prime}$, while $c_{t}=c_{t}^{\prime}$ for all $t \in \mathbb{N}$, such that $t \neq r, s$, then $c^{\prime} \sim c$.

The first axiom is weaker than the standard Pareto Rule, since it postulates the Pareto pre-order only in comparisons of consumption sequences $c$ and $c^{\prime}$ which are eventually identical. The second axiom, which is sometimes also called the Equity Axiom (see Svensson (1980)), embodies a minimal equity principle in that it ensures equal treatment of the consumption of generations over time in the preference structure. ${ }^{11}$

The next axiom incorporates the notion that when we are dealing with "finite horizon intergenerational societies", we have no difficulty in ranking any two consumption sequences. In fact, the axiom makes preferences over such consumption sequences as well-behaved as postulated in the standard theory of numerical representation of preferences, following Debreu (1954, 1959).

In order to formally write this axiom, let us define, for each $T \in \mathbb{N}$,

$$
C(T)=\left\{c \in C: c_{t}=0 \text { for all } t \in \mathbb{N} \text {, satisfying } t>T\right\}
$$

Axiom 3 For each $T \in \mathbb{N}$, the $S W R \succsim$ is a complete pre-order on $C(T)$, and it is continuous in the sum metric on $C(T)$.

This axiom combines Axioms 1 and 2 used by Brock (1970b) in his axiomatic characterization of the overtaking criterion. Note that assumptions like completeness and continuity of the pre-order $\succsim$ on $C(T)$ have been noncontroversial axioms in this literature. It is only when such assumptions are made on the set $C$ that one needs to discuss whether such a complete ranking is always possible, and whether continuity in some topologies on $C$ are more acceptable than others.

Our final axiom is a strong one, but its use is so prevalent in describing intertemporal preferences that we shall have little to say about it, beyond what is already known in the literature. It is the Independence Axiom, and

\footnotetext{
${ }^{11}$ Many authors have felt that a stronger notion than the Anonymity Axiom is needed to reflect intergenerational equity in intertemporal preferences. However, there appears to be general agreement that any notion of intergenerational equity in intertemporal preferences must include the Anonymity Axiom. Since we are attempting an axiomatic characterization of a utilitarian SWR, we feel justified in imposing this weak equity requirement on the SWR.
} 
it follows the postulate, introduced by Debreu (1960) in the finite-horizon context, and by Koopmans (1960) in an infinite-horizon context, in their studies on the representation of preferences by suitable utility functions. ${ }^{12}$

Axiom 4 (Independence) If $c, c^{\prime} \in C$ and $N \in \mathbb{N}$ satisfy:

$$
(c(N), c[N]) \succsim\left(c^{\prime}(N), c[N]\right)
$$

then they must also satisfy:

$$
\left(c(N), c^{\prime}[N]\right) \succsim\left(c^{\prime}(N), c^{\prime}[N]\right)
$$

Remark 1 (i) Loosely speaking, the independence axiom says that the preference between two consumption sequences is independent of those parts of the two streams which are identical, whatever be the vector representing the identical part.

(ii) The above independence axiom is similar to Postulate 3 in Koopmans (1960) and to Postulate P3 in Koopmans, Diamond and Williamson (1964), where it is referred to as "Limited Noncomplementarity".

If a SWR $\succsim$ satisfies the above four axioms, then it is representable by the utilitarian ordering, in comparisons of consumption sequences which are "eventually identical." To state this result formally, we need to introduce the following notation.

Denote by $D$ the subset of $\mathbb{R}^{\mathbb{N}}$, consisting of sequences with at most a finite number of non-zero entries. If $c, c^{\prime} \in C$, then $c$ and $c^{\prime}$ are said to be eventually identical if $\left(c-c^{\prime}\right) \in D$.

Theorem 1 Suppose a SWR $\succsim$ satisfies Axioms 1-4. Then, there is a utility function, $u \in U$, such that for all $c, c^{\prime} \in C$, satisfying $\left(c-c^{\prime}\right) \in D$,

$$
c \succsim c^{\prime} \text { if and only if } \sum_{t=1}^{\infty}\left(u\left(c_{t}\right)-u\left(c_{t}^{\prime}\right)\right) \geq 0
$$

Further, the utility function $u \in U$ satisfying (3) is unique.

\footnotetext{
${ }^{12}$ The independence postulate, developed by Debreu (1960) and Koopmans (1960), follows the pioneering work in this area by Leontief (1947a, 1947b) and Samuelson (1947).
} 
Remark 2 (i)Note that the sum in (3) is well-defined since $c_{t}^{\prime}=c_{t}$ for all but a finite number of periods $t \in \mathbb{N}$.

(ii) In the utilitarian representation of preferences in (3), the fact that the same utility function can be used for all periods follows directly from the Anonymity axiom; in fact, the utility function must be the same for all the periods. The fact that the same utility function can be used regardless of how many non-zero components there are in $\left(c-c^{\prime}\right)$ is a deeper result, involving the Independence axiom in an essential way.

Theorem 1 can be used to provide the following axiomatic characterization of the utilitarian SWR $\succsim_{u}$.

Theorem 2 (i) Suppose there is a utility function, $u \in U$, for which the corresponding utilitarian $S W R \succsim_{u}$ is a subrelation to a $S W R \succsim$. Then $\succsim$ satisfies Axioms 1-4.

(ii) Suppose a $S W R \succsim$ satisfies Axioms 1-4. Then, there is a utility function, $u \in U$, such that the corresponding utilitarian $S W R \succsim_{u}$ is a subrelation to

\subsection{Comparison with the SWR defined by the over- taking criterion}

The standard method of comparing consumption sequences, while respecting the equal treatment of all generations, is by employing the overtaking criterion. The resulting pre-order is a generalization of the one used by Ramsey (1928), and was proposed independently by Atsumi (1965) and von Weizsacker (1965). It was subsequently generalized by Gale (1967), McKenzie (1968) and Brock (1970a). We can formally define it in the following way.

Definition 2 Given a utility function, $u \in U, a$ Ramsey-Atsumi-von Weizsacker (RAV) social welfare relation corresponding to $u$ is a binary relation $R_{u}$, such that for $c, c^{\prime} \in C$,

$$
c R_{u} c^{\prime} \text { iff there is } \bar{N} \in \mathbb{N} \text {, such that } \sum_{t=1}^{N} u\left(c_{t}\right) \geq \sum_{t=1}^{N} u\left(c_{t}^{\prime}\right) \text { for all } N \geq \bar{N}
$$

The symmetric and asymmetric components of $R_{u}$ will be denoted by $I_{u}$ and $P_{u}$. 
Following Brock (1970b), one can obtain an axiomatic characterization of the RAV social welfare relation in terms of Axioms 1-4 of the previous section, and an additional consistency axiom ${ }^{13}$, which we now state.

Axiom 5 (Consistency) For $c, c^{\prime} \in C$,

(a)If there is $N^{\prime} \in \mathbb{N}$, such that $(c(N), 0[N]) \succsim\left(c^{\prime}(N), 0[N]\right)$ for all $N \geq N^{\prime}$, then $c \succsim c^{\prime}$

(b)If there is $N^{\prime} \in \mathbb{N}$, such that $(c(N), 0[N]) \succsim\left(c^{\prime}(N), 0[N]\right)$ for all $N \geq N^{\prime}$, with $(c(N), 0[N]) \succ\left(c^{\prime}(N), 0[N]\right)$ holding for a subsequence of $N \geq N^{\prime}$, then $c \succ c^{\prime}$

Notice that, unlike Axioms 1-4, Axiom 5 does compare consumption sequences which are not eventually identical. Since such comparisons are based on comparisons of consumption sequences which are eventually identical, one might view Axiom 5 also as a continuity restriction on preferences. ${ }^{14}$

The characterization result ${ }^{15}$ can be stated as follows.

Theorem 3 (i)Suppose a $S W R \succsim$ satisfies Axioms 1-5. Then, there is a utility function, $u \in U$, such that the $R A V$ social welfare relation $R_{u}$, corresponding to $u$, is a subrelation to $\succsim$.

(ii) Suppose there is a utility function, $u \in U$, such that the $R A V$ social welfare relation $R_{u}$, corresponding to $u$, is a subrelation to a $S W R \succsim$. Then, $\succsim$ satisfies Axioms 1-5.

The crucial difference between the utilitarian social welfare relation and the RAV social welfare relation is that the latter has to satisfy (in addition to

\footnotetext{
${ }^{13}$ According to Brock (1970), this axiom "captures the notion that decisions on infinite programs are consistent with decisions on finite programs of length $n$ if $n$ is large enough."

${ }^{14}$ Asheim and Tungodden (2004) use an axiom which is similar, and which they view as a continuity restriction.

${ }^{15}$ We present Brock's characterization result in a form so that it may be readily be compared with our results reported in the previous section. Actually, Brock does not impose the Anonymity axiom, and therefore gets a sequence of utility functions (rather than a single one) in his characterization result. He does note however that under an Anonymity axiom, these utility functions would be the same. Brock also does not impose the Pareto axiom, although, to apply the representation theorem of Debreu (1960), he does assume that there are at least three factors of $I^{\mathbb{N}}$, which are essential in the sense of Debreu (1960).
} 
Axioms 1-4) the consistency (or continuity) Axiom 5. This difference might best be explained by looking at an example ${ }^{16}$ of two consumption sequences where, because of Axiom 5, the RAV SWR can compare the two sequences, but where the utilitarian SWR declares them non-comparable.

\section{Example 2}

$$
\left.\begin{array}{lllllll}
c=1 / 2, & 1 / 4, & 1 / 8, & 1 / 16, & 1 / 32, & 1 / 64, & \ldots) \\
c^{\prime}=3 / 4 & 1 / 8, & 1 / 16, & 1 / 32, & 1 / 64, & 1 / 128, & \ldots)
\end{array}\right\}
$$

Given the utility function $u \in U$, which satisfies $u(c)=c$, consider the utilitarian SWR $\succsim_{u}$ and the RAV SWR $R_{u}$ associated with it. We can verify that for $\bar{N}=1$,

$$
\sum_{t=1}^{T} u\left(c_{t}^{\prime}\right)>\sum_{t=1}^{T} u\left(c_{t}\right) \text { for all } T \geq \bar{N}
$$

so that $c^{\prime}$ is preferred to $c$ according to the RAV SWR, corresponding to $u$.

The question arises whether $c^{\prime}$ should be preferred to $c$ by the infinite horizon society. This is not altogether clear. The problems with judging $c^{\prime}$ to be better than $c$ in such a case can be seen as follows.

If we look at any finite-horizon society, and ask the society to rank $c$ versus $c^{\prime}$, they will indeed rank $c^{\prime}$ higher than $c$, if they apply utilitarian principles to themselves. However, no matter how large the finite horizon, there are an infinite number of future generations who rank $c^{\prime}$ below $c$; in fact, all generations beyond the first prefer $c$ to $c^{\prime}$. Thus, it is never possible to have consensus of opinion between any finite horizon society and the infinite number of future generations not included in that finite society. [This should be compared with the "consensus" obtained when the utilitarian SWR can be used to compare consumption sequences].

If one considers the infinite horizon society, one sees that the (infinite) utility sum for both consumption sequences is precisely equal to 1 , and so the RAV SWR in fact violates the utilitarian principles on which it is supposed to be based.

It appears to us that Axiom 5 is not as obvious an axiom to accept as Axioms 1-4 in this context. In other words, we find the RAV SWR less

\footnotetext{
${ }^{16}$ This example is based on the discussion in Basu and Mitra (2003b). The present form of this example owes much to comments on the Basu-Mitra (2003b) paper by Wolfgang Buchholz.
} 
persuasive than the utilitarian SWR. Nevertheless, almost all of the theory of optimal intertemporal allocation, in which generations are treated equally in its preference structure, uses the RAV SWR, and therefore accepts Axiom 5 (in addition to Axioms 1-4). The reason for this is that even though Axiom 5 is not an obvious axiom to accept, it gives sufficient structure to intertemporal preferences so that the theory of optimal intertemporal allocation has some predictive power: a path which is optimal according to this pre-ordering in the typical intertemporal model is unique, and the nature of such an optimal path can be described quite accurately, both in terms of short-run characteristics (the Ramsey-Euler or competitive conditions), and long-run behavior (the turnpike property).

The presumption appears to be that if we wanted to proceed with intertemporal preferences satisfying only Axioms 1-4 (that is, without imposing something like Axiom 5), one would not have a useful theory of optimal behavior over time. In principle, there could be many maximal points according to the utilitarian SWR, and it might be the case that their behavior (as a group of paths) fails to have some unifying mode worth characterizing. Unfortunately, this issue has not been explored in the literature, and therefore such misgivings about a theory based solely on the utilitarian SWR might be premature. ${ }^{17}$

We will establish the rather surprising result that in the standard forestry model, a maximal point according to the utilitarian $S W R$ is in fact unique and coincides with the optimal point according to the RAV SWR. This shows that we do not need the SWR induced by the overtaking criterion, and consequently the comparisons entailed by Axiom 5, to present the theory of forest management, respecting intergenerational equity. This puts the theory on a more robust and satisfactory basis in terms of the postulated intertemporal preference structure.

\subsection{Intertemporal Inequality Aversion}

In order to obtain the result mentioned above, in the standard forestry model, we need a bit more structure on the utility function derived in Theorem 1, based on Axioms 1-4. Specifically, we will need the utility function to be concave, and strictly mid-concave, on $I$. While this is standard fare for many

\footnotetext{
${ }^{17}$ In this connection, one might note that in a recent paper, Asheim and Buchholz (2004) work with a social preference relation, which has even less structure than ours; the SWR in their paper is restricted only to satisfy the Pareto and Anonymity axioms.
} 
models of intertemporal allocation, the axiomatic basis of this requirement is often not stressed. ${ }^{18}$ We present an exposition of this aspect of the theory by imposing the following additional axiom on preferences.

Axiom 6 (Strong Convexity ) For $c, c^{\prime} \in C$ with $\left(c-c^{\prime}\right) \in D$, and $c \neq c^{\prime}$,

$$
c \sim c^{\prime} \text { implies }\left(\frac{1}{2}\right) c+\left(\frac{1}{2}\right) c^{\prime} \succ c
$$

The terminology "strong convexity" of preferences follows Debreu (1959, p.61). ${ }^{19}$ The standard way to interpret this axiom is that the underlying preference structure exhibits inequality aversion, where the inequality in question here is with respect to the intertemporal consumption pattern.

A function $u \in U$ will be called concave if for all $x, x^{\prime} \in L$ and $\lambda \in(0,1)$, we have $u\left(\lambda x+(1-\lambda) x^{\prime}\right) \geq \lambda u(x)+(1-\lambda) u\left(x^{\prime}\right)$. It will be called strictly mid-concave if for all $x, x^{\prime} \in L$ with $x \neq x^{\prime}$,

$$
u\left(\left(\frac{1}{2}\right) x+\left(\frac{1}{2}\right) x^{\prime}\right)>\left(\frac{1}{2}\right) u(x)+\left(\frac{1}{2}\right) u\left(x^{\prime}\right)
$$

We define the class of utility functions $U^{c}=\{u \in U: u$ is concave and strictly mid-concave on $L\}$.

Theorem 4 Suppose a $S W R \succsim$ satisfies Axioms 1-4 and 6. Then, there is a utility function, $u \in U^{c}$, such that for all $c, c^{\prime} \in C$, satisfying $\left(c-c^{\prime}\right) \in D$,

$$
c \succsim c^{\prime} \text { if and only if } \sum_{t=1}^{\infty}\left(u\left(c_{t}\right)-u\left(c_{t}^{\prime}\right)\right) \geq 0
$$

Further, the utility function $u \in U^{c}$ satisfying (5) is unique.

\section{A Forestry Model}

The standard model of forest management under intertemporal equity can be described in terms of the objects $(f, A, b, u)$, where $(f, A, b)$ represent the

\footnotetext{
${ }^{18}$ See Yaari (1977) for the general result relating convexity of preference relations to concavity of utility functions, when the preference relation has an additively separable utility representation.

${ }^{19}$ Actually, Debreu asserts the strict preference for all non-trivial convex-combinations of $c$ and $c^{\prime}$. We will need the axiom only in its "strictly mid-convex" form.
} 
technological aspects, and $u$ represents the preferences ${ }^{20}$. We describe each in turn in what follows.

We begin with $f$, a production function from $\mathbb{R}_{+}$to $\mathbb{R}_{+}$, which relates the timber content of a tree $f(a)$ to the age of the tree, $a$. The following assumptions on $f$ are maintained:

(A.1) There is $\tilde{a} \geq 1$, such that $f(a)=0$ for $a \in[0, \tilde{a}]$.

(A.2) $f$ is continuous for $a \geq \tilde{a}$, and there is a positive integer $n>a$, such that (i) $f$ is increasing for $a \in[\tilde{a}, n]$, and (ii) $f$ is decreasing for $a>n$.

(A.3) There is a positive integer $m$, satisfying $\tilde{a}<m<n$, such that (i) $[f(a) / a]$ is maximized at $a=m$ among all $a \in\{1, \ldots, n\}$, and (ii) $[f(a) / a]<$ $[f(m) / m]$ for all $a \in\{1, \ldots, n\}$, with $a \neq n$.

We will denote the vector $(f(1), \ldots, f(n))$ by $P$; note that $P \in \mathbb{R}_{+}^{n}$. In what follows, we normalize $f(n)=1$ by choice of units in which the timber content is measured.

Next, we describe $(A, b)$, which indicate the transition possibilities of forestry land occupied by trees of different ages. It is useful, in this connection, to indicate the nature of evolution of the forest informally, before introducing the matrix-vector notation.

Given (A.2), for any reasonable objective function of the forest manager, trees will never be allowed to grow beyond age $n$. We therefore take this as a condition of feasibility itself. The total land available for forestry is taken to be one unit (by appropriate choice of units in which land is measured). Imagine that we are starting at the end of time period 1 with a standing forest, which might be described by the land occupied by output of trees of ages 1 to $n$. This will be written as $\left(y_{1}^{1}, \ldots, y_{1}^{n}\right)$. At the end of time period 1, two things are supposed to happen instantaneously, by the nature of our point-input, point-output framework. First trees of different ages are harvested. Second, new seedlings (trees of age 0 ) are planted in the cleared areas. The land released by harvests are described by $\left(h_{1}^{1}, \ldots, h_{1}^{n}\right)$. Since trees are never allowed to grow beyond age $n$, we must have $h_{1}^{n}=y_{1}^{n}$. The land occupied by input of trees of various ages at the end of time period 1 can then be described by $\left(x_{1}^{0}, \ldots, x_{1}^{n-1}, x_{1}^{n}\right)$, where $\left(y_{1}^{1}, \ldots, y_{1}^{n}\right)-\left(h_{1}^{1}, \ldots, h_{1}^{n}\right)=\left(x_{1}^{1}, \ldots, x_{1}^{n-1}, x_{1}^{n}\right)=\left(x_{1}^{1}, \ldots, x_{1}^{n-1}, 0\right)$, and $x_{1}^{0}=h_{1}^{1}+\cdots+h_{1}^{n}$.

\footnotetext{
${ }^{20}$ In terms of notation (but not in terms of content) the present version differs slightly from that used in Mitra and Wan (1986). References to the earlier literature, on which the model is based, can be found in Mitra and Wan (1986).
} 
The trees grow during time period 2, with trees of age $a \in\{0, \ldots, n-1\}$ at the end of time period 1, becoming trees of age $a+1$ at the end of time period 2. Thus, the land occupied by output of trees at the end of time period 2 is $\left(y_{2}^{1}, \ldots, y_{2}^{n}\right)=\left(x_{1}^{0}, \ldots, x_{1}^{n-1}\right)$. The above process is now repeated indefinitely.

This informal description can be formalized as follows. Define the $n \times n$ matrix $A$ and the vector $b \in \mathbb{R}^{n}$ as:

$$
A=\left[\begin{array}{llllll}
0 & 1 & 0 & 0 & \ldots & 0 \\
0 & 0 & 1 & 0 & \ldots & 0 \\
\ldots & \ldots & \ldots & \ldots & \ldots & \ldots \\
0 & 0 & 0 & 0 & \ldots & 1 \\
0 & 0 & 0 & 0 & \ldots & 0
\end{array}\right]=\left[\begin{array}{ll}
0_{n} & I_{n-1} \\
0 & 0_{n}
\end{array}\right] ; b=(1,1, \ldots, 1)
$$

Denote the set $\left\{x \in \mathbb{R}_{+}^{n}: b x=1\right\}$ by $Q$, and define a transition possibility set by:

$$
\Omega=\left\{(y, z) \in Q^{2}: A z \leq y\right\}
$$

A path starting from $y \in Q$ is a sequence $\left(y_{t}\right)_{1}^{\infty}=\left(y_{t}^{1}, \ldots, y_{t}^{n}\right)_{1}^{\infty}$ satisfying:

$$
\left(y_{t}, y_{t+1}\right) \in \Omega \text { for } t \in \mathbb{N} \text {, and } y_{0}=y .
$$

We can associate with a path $\left(y_{t}\right)_{1}^{\infty}$ a sequence $\left(h_{t}\right)_{1}^{\infty}=\left(h_{t}^{1}, \ldots, h_{t}^{n}\right)_{1}^{\infty}$ defined by:

$$
h_{t}=y_{t}-A y_{t+1} \text { for } t \in \mathbb{N}
$$

and a sequence $\left(x_{t}\right)_{1}^{\infty}=\left(x_{t}^{0}, x_{t}^{1}, \ldots, x_{t}^{n}\right)_{1}^{\infty}$ defined by:

$$
\left(x_{t}^{0}, x_{t}^{1}, \ldots, x_{t}^{n}\right)=\left(1-\left\|A y_{t+1}\right\|, A y_{t+1}\right) \text { for } t \in \mathbb{N}
$$

Note that $h_{t} \in \mathbb{R}_{+}^{n}$, with $h_{t}^{n}=y_{t}^{n}$ for $t \in \mathbb{N}$, and $x_{t} \in \mathbb{R}_{+}^{n+1}$, with $x_{t}^{n}=0$ and $\left\|x_{t}\right\|=1$ for $t \in \mathbb{N}$.

The timber content obtained by harvest $h_{t}$ is $P h_{t}$. So, we can associate with a path $\left(y_{t}\right)_{1}^{\infty}$, the consumption sequence $\left(c_{t}\right)_{1}^{\infty}$ defined by:

$$
c_{t}=P h_{t} \text { for } t \in \mathbb{N}
$$

Notice that (by choice of $f(n)=1$ ), we must have $c_{t} \in[0,1]$ for $t \in \mathbb{N}$.

Finally, we turn to preferences defined on consumption sequences. We will suppose that there is a SWR $\succsim$ satisfying Axioms 1-4 and 6, so there is a unique utility function, $u \in U^{c}$, as described in Theorem 4 of Section 3. 
A path $\left(y_{t}^{\prime}\right)_{1}^{\infty}$ from $y \in Q$ dominates a path $\left(y_{t}\right)_{1}^{\infty}$ from $y \in Q$ if the associated consumption sequences $\left(c_{t}^{\prime}\right)_{1}^{\infty}$ and $\left(c_{t}\right)_{1}^{\infty}$ satisfy the condition that $\left(c_{t}^{\prime}\right)_{1}^{\infty} \succ_{u}\left(c_{t}\right)_{1}^{\infty}$ [where the SWR $\succsim_{u}$, as defined by (U) of Section 3, corresponds to the $u \in U^{c}$, mentioned above]. That is,

$$
\exists N \in \mathbb{N} \text { such that } c^{\prime}[N]=c[N] \text { and } \sum_{t=1}^{N} u\left(c_{t}^{\prime}\right)>\sum_{t=1}^{N} u\left(c_{t}\right)
$$

A path $\left(y_{t}\right)_{1}^{\infty}$ is called maximal if there is no path $\left(y_{t}^{\prime}\right)_{1}^{\infty}$ from $y$ which dominates it.

In words, a maximal path is a path, such that there is no path (from the same initial conditions) which is better in terms of the social welfare relation $\succsim_{u}$. It is, perhaps, worth emphasizing that this does not necessarily mean that given any path (from the same initial conditions), a maximal path is at least as good as the given path; in fact, the two paths need not be comparable. Thus, the definition of maximality (like the definition of Pareto efficiency) allows for multiple maximal points on the feasible set, given the pre-order. ${ }^{21}$

\section{Maximum Sustained Yield Forest}

Suppose the forest does not change at all from one period to the next. What is the "best" composition of the forest in that case ? This is what is meant when one speaks of the forest with the maximum sustained yield. In the terminology of intertemporal allocation theory, one is concerned here with the "golden-rule" forest.

We show that there is a unique golden-rule forest, and obtain dual variables (shadow prices) which provide "price support" to such a forest. These shadow prices are in fact prices (expressed in terms of the utility good) of the various subplots of land, according to the age of trees standing on each subplot. The golden rule prices maximize "generalized profit" [the utility plus the valuation of terminal subplots of land minus the valuation of initial plots of land, terminal and initial referring to a unit time period] at the golden-rule forest among all possible activities in the transition possibility set.

\footnotetext{
${ }^{21}$ It will turn out, as noted later in Section 8 , that there is actually a unique maximal path (from given initial conditions) in the model we are analyzing. But, this is a result of the notion of maximality applied to the standard forestry model; it is not definitional.
} 
We go on to show (in Proposition 1) that those points in the transition possibility set which stay away (uniformly) from the golden-rule must suffer a (uniform) loss of "generalized profit" (compared to the golden-rule forest), when evaluated at the golden-rule prices.

To discuss the golden-rule, let us introduce the welfare function, $w: \Omega \rightarrow$ $L$, defined by:

$$
w(y, z)=u(P(y-A z)) \text { for all }(y, z) \in \Omega
$$

A golden-rule forest is a vector $\bar{y} \in Q$ which solves the problem:

$$
\left.\begin{array}{ll}
\text { Max } & u(P(y-A y)) \\
\text { subject to } & (y, y) \in \Omega
\end{array}\right\}(G R)
$$

Since $u$ is increasing, the above problem is the same as:

$$
\left.\begin{array}{ll}
\text { Max } & P(y-A y) \\
\text { subject to } & (y, y) \in \Omega
\end{array}\right\}\left(G R^{\prime}\right)
$$

To characterize the golden-rule, note that for $(y, y) \in \Omega$, we have $y_{i} \geq y_{i+1}$ for $i \in\{1, \ldots, n-1\}$. So, we get:

$$
\begin{aligned}
P(y-A y)= & P_{1}\left(y_{1}-y_{2}\right)+\cdots+P_{n-1}\left(y_{n-1}-y_{n}\right)+P_{n} y_{n} \\
= & P_{1}\left(y_{1}-y_{2}\right)+\left[P_{2} / 2\right] 2\left(y_{2}-y_{3}\right)+\cdots \\
& +\left[P_{n-1} /(n-1)\right](n-1)\left(y_{n-1}-y_{n}\right)+\left[P_{n} / n\right] n y_{n} \\
\leq & {\left[P_{m} / m\right]\left[\left(y_{1}-y_{2}\right)+2\left(y_{2}-y_{3}\right)+\cdots+(n-1)\left(y_{n-1}-y_{n}\right)+n y_{n}\right.} \\
= & {\left[P_{m} / m\right]\left[y_{1}+y_{2}+\cdots+y_{n-1}+y_{n}\right] } \\
= & {\left[P_{m} / m\right] }
\end{aligned}
$$

the inequality following from assumption $(\mathrm{A} .3)(\mathrm{i})$ on $f$. Defining $\bar{y}=[(1 / m), \ldots,(1 / m), 0, \ldots, 0]$ in $Q$, it follows that $\bar{y}$ is a golden-rule forest.

By (A.3)(ii), a strict inequality is produced in the above calculation, unless $y_{i}-y_{i+1}=0$ for all $i \in\{1, \ldots, n-1\}$, with $i \neq m$ and $y_{n}=$ 0 . Thus, if $y$ is a golden-rule forest, then $y=\left(y_{m}, \ldots, y_{m}, 0, \ldots, 0\right)$, and since $y \in Q$, we must in fact have $y=[(1 / m), \ldots,(1 / m), 0, \ldots, 0]$. Thus, $\bar{y}=[(1 / m), \ldots,(1 / m), 0, \ldots, 0]$ is the unique golden-rule forest. Clearly, the consumption associated with a golden-rule forest is stationary, and equal to $\left[P_{m} / m\right]$; we will denote it by $\bar{c}$. 
A convenient "price support property" of the golden-rule may now be noted. Define:

$$
q=\bar{c}(1, \ldots, n) \text { and } p=u_{+}^{\prime}(\bar{c}) q
$$

where $u_{+}^{\prime}(\bar{c})$ is the right-hand derivative of $u$ at $\bar{c}$. Then, we have:

$$
u(P(y-A z))+p z-p y \leq u(\bar{c}) \text { for all }(y, z) \in \Omega
$$

To see this, note that $P \leq q$ by (A.3). Thus, for $(y, z) \in \Omega$, we have:

$$
P(y-A z) \leq q(y-A z)=q y-q z+q(z-A z)
$$

Now, $(q-q A)=\bar{c}(1,2, \ldots, n)-\bar{c}(0,1, \ldots, n-1)=\bar{c}(1, \ldots, 1,1)$, so $q(z-$ $A z)=(q-q A) z=\bar{c}\|z\|=\bar{c}$. Thus, (7) yields:

$$
P(y-A z) \leq q y-q z+\bar{c} \text { for all }(y, z) \in \Omega
$$

Using the concavity of $u$, we have:

$$
u(P(y-A z))-u(\bar{c}) \leq u_{+}^{\prime}(\bar{c})[P(y-A z)-\bar{c}] \leq u_{+}^{\prime}(\bar{c})[q y-q z]
$$

the last inequality in (9) following from (8). Using $p=u_{+}^{\prime}(\bar{c}) q$ and rearranging terms in (9) yields (6). We will refer to the $p$ defined in (GRP) as the golden-rule price.

Following McKenzie (1968), let us define the value loss of operating at $(y, z) \in \Omega$ as:

$$
\delta(y, z)=u(\bar{c})-[u(P(y-A z))+p z-p y]
$$

Clearly, $\delta(y, z) \geq 0$ for all $(y, z) \in \Omega$, and $\delta(\bar{y}, \bar{y})=0$.

The points which have zero value-loss have a very special structure, as we note in the following result ${ }^{22}$. For this purpose, we denote the vector $(1 / m) e^{m}$ [where $e^{m}$ is the $m-t h$ unit vector in $\mathbb{R}^{n}$ ] by $g$.

Proposition 1 (i) If $(y, z) \in \Omega$ and $\delta(y, z)=0$, then:

$$
(y-A z)=g
$$

(ii) Given $\varepsilon>0$, there is $\delta>0$, such that if $(y, z) \in \Omega$, and $d((y-$ $A z), g) \geq \varepsilon$, then $\delta(y, z) \geq \delta$.

\footnotetext{
${ }^{22}$ The second part of this Proposition is a result along the lines of the well-known "valueloss lemma", due to Radner (1961), Atsumi (1965), and McKenzie (1968).
} 
Proof. (i) Note that the inequality in (6) depends on the inequalities in (7) and (9). Using (7), we can see that in order to have $\delta(y, z)=0$, we must have:

$$
y_{i}-z_{i+1}=0 \text { for all } i \in\{1, \ldots, n-1\}, i \neq m \text {, and } y_{n}=0
$$

Thus, $(y-A z)$ must be of the form $\left(0,0, \ldots, y_{m}-z_{m+1}, 0, \ldots, 0\right)$. Thus, we must have $P(y-A z)=P_{m}\left(y_{m}-z_{m+1}\right)$. Denoting $P_{m}\left(y_{m}-z_{m+1}\right)$ by $c$, we see from the left-hand inequality in (9),

$$
u(c)-u(\bar{c}) \leq u_{+}^{\prime}(\bar{c})[c-\bar{c}]
$$

In order to have $\delta(y, z)=0$, we must have equality in (12).

If $c \neq \bar{c}$, then defining $c^{\prime}=\left(\frac{1}{2}\right) c+\left(\frac{1}{2}\right) \bar{c}$, we have by concavity of $u$, and strict mid-concavity of $u$,

$$
\left(\frac{1}{2}\right)[u(c)-u(\bar{c})]=\left(\frac{1}{2}\right) u(c)+\left(\frac{1}{2}\right) u(\bar{c})-u(\bar{c})<u\left(c^{\prime}\right)-u(\bar{c}) \leq u_{+}^{\prime}(\bar{c})\left[c^{\prime}-\bar{c}\right]=\left(\frac{1}{2}\right) u_{+}^{\prime}(\bar{c})[c-\bar{c}]
$$

and we cannot have equality in (12). Thus, for equality in (12), we must have $c=\bar{c}$. Thus, $\left(y_{m}-z_{m+1}\right)=(1 / m)$, so that $(y-A z)=g$.

(ii) Suppose, on the contrary, there is a sequence $\left(y^{s}, z^{s}\right) \in \Omega$ with $s \in \mathbb{N}$, such that $d\left(\left(y^{s}-A z^{s}\right), g\right) \geq \varepsilon$, but $\delta\left(y^{s}, z^{s}\right) \rightarrow 0$ as $s \rightarrow \infty$. Since $\Omega$ is compact, there is a subsequence $\left(y^{s^{\prime}}, z^{s^{\prime}}\right)$ of $\left(y^{s}, z^{s}\right)$, converging to $(\hat{y}, \hat{z}) \in \Omega$. Since $d\left(\left(y^{s^{\prime}}-A z^{s^{\prime}}\right), g\right) \geq \varepsilon$, we must have $d((\hat{y}-A \hat{z}), g) \geq \varepsilon$. By (11), we must have $\delta(\hat{y}, \hat{z})>0$.

Since $\left(y^{s^{\prime}}, z^{s^{\prime}}\right)$ converges to $(\hat{y}, \hat{z})$, we must have $\delta\left(y^{s^{\prime}}, z^{s^{\prime}}\right)$ converging to $\delta(\hat{y}, \hat{z})$, by $(10)$ and the continuity of $u$. Since $\delta\left(y^{s^{\prime}}, z^{s^{\prime}}\right) \rightarrow 0$, we have $\delta(\hat{y}, \hat{z})=$ 0 , a contradiction to the result obtained in the previous paragraph.

\section{Long-Run Properties of Maximal Paths}

In this section, we establish the following long-run property of maximal paths: there is asymptotic convergence to the maximum sustained yield forest, starting from an arbitrary initial forest. This provides a theoretical justification for focusing on the maximum sustained yield forest as a goal of forestry policy.

We show that from every initial forest, one can reach the steady-state of the golden-rule forest in a finite number of periods (Lemma 1). Thus, there 
are always paths which suffers finite value losses (in terms of the sum of generalized profits evaluated at the golden-rule prices) compared to the golden-rule forest, over the infinite horizon. Such paths are called "good paths", and a maximal path is shown (in Proposition 2) to be a good path. Because activities which stay away uniformly from the golden rule must suffer uniform per period value loss (Proposition 1 in the previous section), paths which stay away uniformly from the golden-rule for an infinite number of periods must suffer infinite value losses. Thus good paths (which, by definition, suffer only finite value losses) must exhibit asymptotic convergence to the golden-rule. Consequently, maximal paths must also exhibit this property, since they are good paths (Theorem 5). ${ }^{23}$

The principal technical result needed for our analysis is the ability to move from any initial forest to any other forest in a finite number of periods, independent of the initial and terminal compositions. In fact, we show that this transition can always be made in $(n+1)$ periods.

Lemma 1 Given any $(y, \hat{y}) \in Q^{2}$, there exist $\left(z_{1}, \ldots, z_{n+2}\right)$ such that:

$$
\text { (i) }\left(z_{s}, z_{s+1}\right) \in \Omega \text { for } s=1, \ldots, n+1 ;(i i) z_{1}=y, z_{n+2}=\hat{y} \text {. }
$$

Proof. The proof consists of simply defining $\left(z_{1}, \ldots, z_{n+2}\right)$ appropriately and checking that it satisfies (13). Let us define $\left(z_{1}, \ldots, z_{n+2}\right)$ as follows:

$$
\left.\begin{array}{l}
z_{1}=y \\
z_{2}=(1,0, \ldots, 0) \\
z_{3}=\left(\hat{y}^{n}, 1-\hat{y}^{n}, 0, \ldots, 0\right) \\
z_{4}=\left(\hat{y}^{n-1}, \hat{y}^{n}, 1-\hat{y}^{n}-\hat{y}^{n-1}, 0, \ldots, 0\right) \\
\ldots \quad \cdots \\
z_{n+1}=\left(\hat{y}^{2}, \hat{y}^{3}, \ldots, \hat{y}^{n}, \hat{y}^{1}\right) \\
z_{n+2}=\hat{y}
\end{array}\right\}
$$

It is straightforward to verify from this definition that (13) holds.

Following Gale (1967), let us call a path $\left(y_{t}\right)_{1}^{\infty}$ good if there is a real number $\bar{G}$ such that:

$$
\sum_{t=1}^{T}\left[u\left(P\left(y_{t}-A y_{t+1}\right)\right)-u(\bar{c})\right] \geq \bar{G} \text { for all } T \in \mathbb{N}
$$

\footnotetext{
${ }^{23}$ For an excellent non-technical discussion of the methods used in Mitra and Wan (1986), which parallel the methods used in this section (as well as the previous one) see the recent paper by M. Ali Khan (2004).
} 
Informally, a good path is one which is at most finitely worse that the goldenrule path in terms of partial sums of its utlities.

We now show, using Lemma 1, that a maximal path is necessarily good, so that it inherits all the well-known long-run properties of good paths.

Proposition 2 Let $\left(y_{t}\right)_{1}^{\infty}$ be a maximal path from $y \in Q$. Then $\left(y_{t}\right)_{1}^{\infty}$ is good.

Proof. Define $G=-2(n+1) u(\bar{c})$. We claim that for all $T>2(n+2)$, we have:

$$
\sum_{t=1}^{T}\left[u\left(P\left(y_{t}-A y_{t+1}\right)\right)-u(\bar{c})\right] \geq G
$$

Suppose, on the contrary, there is some $T \in \mathbb{N}$, such that $T>2(n+2)$, and

$$
\sum_{t=1}^{T}\left[u\left(P\left(y_{t}-A y_{t+1}\right)\right)-u(\bar{c})\right]<G
$$

Since $(y, \bar{y}) \in Q^{2}$, we can use Lemma 1 to obtain $\left(z_{1}, \ldots, z_{n+2}\right)$ such that:

$$
\text { (i) }\left(z_{s}, z_{s+1}\right) \in \Omega \text { for } s=1, \ldots, n+1 ;(i i) z_{1}=y, z_{n+2}=\bar{y} \text {. }
$$

Since $\left(\bar{y}, y_{T+1}\right) \in Q^{2}$, we can use Lemma 1 to obtain $\left(z_{1}^{\prime}, \ldots, z_{n+2}^{\prime}\right)$ such that:

$$
\text { (i) }\left(z_{s}^{\prime}, z_{s+1}^{\prime}\right) \in \Omega \text { for } s=1, \ldots, n+1 ;(i i) z_{1}^{\prime}=\bar{y}, z_{n+2}^{\prime}=y_{T+1} \text {. }
$$

Define a sequence $\left(y_{t}^{\prime}\right)_{1}^{\infty}$ as follows:

$$
\begin{array}{ll}
y_{t}^{\prime}=z_{t} & \text { for } t=1, \ldots, n+2 \\
y_{t}^{\prime}=\bar{y} & \text { for } t=n+3, \ldots, T-(n+1) \\
y_{t}^{\prime}=z_{t-(T-n-1)}^{\prime} & \text { for } t=T-n, \ldots, T+1 \\
y_{t}^{\prime}=y_{t} & \text { for } t>T+1
\end{array}
$$

Using (16), (17) and (18) it is easy to check that $\left(y_{t}^{\prime}\right)_{1}^{\infty}$ is a path from $y \in Q$. Since $y_{t}^{\prime}=y_{t}$ for $t \geq T+1$, we have $c_{t}^{\prime}=c_{t}$ for $t \geq T+1$, and $c_{t}^{\prime}=\bar{c}$ for $t=n+3, \ldots, T-(n+1)$. Using (18), and $u \in U$, it follows that:

$$
\sum_{t=1}^{T}\left[u\left(P\left(y_{t}^{\prime}-A y_{t+1}^{\prime}\right)\right)-u(\bar{c})\right] \geq-2(n+1) u(\bar{c})=G
$$


Thus, using (15) and (19), we have:

$$
\sum_{t=1}^{T} u\left(P\left(y_{t}^{\prime}-A y_{t+1}^{\prime}\right)\right)>\sum_{t=1}^{T} u\left(P\left(y_{t}-A y_{t+1}\right)\right)
$$

But, this implies that $\left(y_{t}\right)_{1}^{\infty}$ is not maximal, a contradiction. Thus, our claim (14) must hold, and it follows that $\left(y_{t}\right)_{1}^{\infty}$ is good.

Using the above proposition, we can now summarize the long-run properties of maximal paths in the following theorem ${ }^{24}$. To this end, given a path $\left(y_{t}\right)_{1}^{\infty}$ from $y \in Q$, we associate with it a value-loss sequence $\left(\delta_{t}\right)_{1}^{\infty}$ defined by:

$$
\delta_{t}=\delta\left(y_{t}, y_{t+1}\right) \text { for } t \in \mathbb{N}
$$

Theorem 5 Let $\left(y_{t}\right)_{1}^{\infty}$ be a maximal path from $y \in Q$. Then, we have:

$$
\begin{gathered}
\sum_{t=1}^{\infty} \delta_{t}<\infty \\
\delta_{t} \rightarrow 0 \text { as } t \rightarrow \infty \\
d\left(\left(y_{t}-A y_{t+1}\right), g\right) \rightarrow 0 \text { as } t \rightarrow \infty \\
d\left(y_{t}, \bar{y}\right) \rightarrow 0 \text { as } t \rightarrow \infty
\end{gathered}
$$

Proof. Given any $T \in \mathbb{N}$, we can use (6) to write:

$$
\begin{aligned}
\sum_{t=1}^{T}\left[u\left(P\left(y_{t}-A y_{t+1}\right)\right)-u(\bar{c})\right] & =\sum_{t=1}^{T}\left[p y_{t}-p y_{t+1}\right]-\sum_{t=1}^{T} \delta_{t} \\
& =\left[p y-p y_{T+1}\right]-\sum_{t=1}^{T} \delta_{t}
\end{aligned}
$$

Since $\left(y_{t}\right)_{1}^{\infty}$ is maximal, it is good by Proposition 2 , so there is $G \in \mathbb{R}$ such that:

$$
\sum_{t=1}^{T}\left[u\left(P\left(y_{t}-A y_{t+1}\right)\right)-u(\bar{c})\right] \geq G \text { for all } T \in \mathbb{N}
$$

\footnotetext{
${ }^{24}$ The analysis follows Mitra and Wan (1986) closely.
} 
Using (24) and (25), we obtain:

$$
\sum_{t=1}^{T} \delta_{t} \leq p y-G \leq n-G \text { for all } T \in \mathbb{N}
$$

Since $\delta_{t} \geq 0$ by (6), (26) establishes (20). Clearly, (21) follows immediately from (20). Using Proposition 1 and (21), we obtain (22).

We now verify (23) as follows. Using (22), given any $\varepsilon>0$, we can choose $T \in \mathbb{N}$, such that:

$$
d\left(\left(y_{t}-A y_{t+1}\right), g\right)<\left(\varepsilon / n^{4}\right) \text { for } t \geq T
$$

We will show that:

$$
d\left(y_{s+m}, \bar{y}\right) \leq \varepsilon \text { for all } s>T
$$

Using (27), we note that for each $t \geq T$, we have:

$$
\left.\begin{array}{l}
\text { (a) } y_{t}^{i}-y_{t+1}^{i+1}<\left(\varepsilon / n^{4}\right) \text { for } i=1, \ldots, m-1, m+1, \ldots, n \\
\text { (b) } y_{t}^{m}-y_{t+1}^{m+1}>(1 / m)-\left(\varepsilon / n^{4}\right)
\end{array}\right\}
$$

Then, for $t>T$, we must have:

$$
y_{t+1}^{1}=\sum_{i=1}^{n} h_{t}^{i}=\sum_{i=1}^{n}\left(y_{t}^{i}-y_{t+1}^{i+1}\right)>(1 / m)-\left(\varepsilon / n^{3}\right)
$$

And, for $j=1, \ldots, m-1$ and $t>T$,

$$
\begin{aligned}
y_{t+j+1}^{j+1} & =y_{t+1}^{1}+\sum_{i=1}^{j}\left(y_{t+i+1}^{i+1}-y_{t+i}^{i}\right) \\
& >(1 / m)-\left(\varepsilon / n^{3}\right)-r\left(\varepsilon / n^{4}\right) \\
& \geq(1 / m)-\left(\varepsilon / n^{2}\right)
\end{aligned}
$$

Using (30) and (31), we have for $t>T$,

$$
y_{t+j}^{j}>(1 / m)-\left(\varepsilon / n^{2}\right) \text { for } j=1, \ldots, m
$$

Now pick any $s>T$. Then, using (32), we have:

$$
\sum_{i=m+1}^{n} y_{s+m}^{i}<1-m\left[(1 / m)-\left(\varepsilon / n^{2}\right)\right]=m \varepsilon / n^{2}
$$


Also, using (32), we claim that:

$$
y_{s+m}^{i}<(1 / m)+(\varepsilon / n) \text { for } i=1, \ldots, m
$$

For if $y_{s+m}^{i} \geq(1 / m)+(\varepsilon / n)$ for some $i \in\{1, \ldots, m\}$, then:

$$
\sum_{i=1}^{n} y_{s+m}^{i} \geq \sum_{i=1}^{m} y_{s+m}^{i}>(1 / m)+(\varepsilon / n)+(m-1)\left[(1 / m)-\left(\varepsilon / n^{2}\right)\right] \geq 1
$$

which contradicts the fact that $y_{s+m} \in Q$. This establishes our claim (34). Now, using (32) and (34), we have:

$$
\left|y_{s+m}^{i}-(1 / m)\right|<(\varepsilon / n) \text { for } i=1, \ldots, m
$$

Using (33) and (35), and $m \leq(n-1)$, we have for $s>T$,

$$
\begin{aligned}
d\left(y_{s+m}, \bar{y}\right) & =\sum_{i=1}^{m}\left|y_{s+m}^{i}-(1 / m)\right|+\sum_{i=m+1}^{n} y_{s+m}^{i} \\
& <m(\varepsilon / n)+m\left(\varepsilon / n^{2}\right) \\
& \leq(n-1) \varepsilon\left[(1 / n)+\left(1 / n^{2}\right)\right] \\
& =\varepsilon\left(n^{2}-1\right) / n^{2}<\varepsilon
\end{aligned}
$$

This establishes (28) and therefore (23).

\section{Shadow Prices for Maximal Paths}

Our investigation of the forestry model so far has shown that its central results under intergenerational equity can be obtained without any reference to the overtaking criterion. Our notion of maximality, based completely on ranking of paths which are eventually identical, suffices for this purpose.

However, in order to tie up our present study with the earlier study by Mitra and Wan (1986), we need to investgate the relation of maximal paths to paths which are "optimal" according to the overtaking criterion, a precise definition of which is given below.

We continue to suppose that there is a SWR $\succsim$ satisfying Axioms 1-4 and 6 , so there is a unique utility function, $u \in U^{c}$, as described in Theorem 4 of Section 3. 
A path $\left(y_{t}^{\prime}\right)_{1}^{\infty}$ from $y \in Q$ weakly overtakes a path $\left(y_{t}\right)_{1}^{\infty}$ from $y \in Q$ if the associated consumption sequences $\left(c_{t}^{\prime}\right)_{1}^{\infty}$ and $\left(c_{t}\right)_{1}^{\infty}$ satisfy the condition that $\left(c_{t}^{\prime}\right)_{1}^{\infty} P_{u}\left(c_{t}\right)_{1}^{\infty}$ [where the SWR $R_{u}$ as defined by (R) in Section 3, corresponds to the $u \in U^{c}$ mentioned above]. This will be the case if and only if there is $\bar{N} \in \mathbb{N}$, such that:

$$
\sum_{t=1}^{N} u\left(c_{t}^{\prime}\right) \geq \sum_{t=1}^{N} u\left(c_{t}\right) \text { for all } N \geq \bar{N}
$$

and :

$$
\sum_{t=1}^{N^{\prime}} u\left(c_{t}^{\prime}\right)>\sum_{t=1}^{N^{\prime}} u\left(c_{t}\right) \text { for a subsequence } N^{\prime} \text { of } N \geq \bar{N}
$$

A path $\left(y_{t}\right)_{1}^{\infty}$ from $y \in Q$ is called optimal if there is no path $\left(y_{t}^{\prime}\right)_{1}^{\infty}$ from $y$ which weakly overtakes it.

In words, an optimal path is a path, such that there is no path (from the same initial conditions) which is better in terms of the social welfare relation $R_{u}$. It is worth noting, by comparing the definitions of maximality and optimality, that an optimal path is necessarily maximal.

This brings us to the part of our paper which distinguishes the present analysis of the forest management problem from the one offered in Mitra and Wan (1986). This distinction parallels the difference in approaches used by Brock (1970a) on the one hand, and by Gale (1967) and McKenzie (1968) on the other, in developing the general theory of optimal intertemporal allocation under the overtaking criterion.

Brock (1970a) showed that the theory of optimal intertemporal allocation under the overtaking criterion can be developed fully without finding shadow prices to support infinite-horizon paths. One only needs the price-support property of the golden-rule, a technically simple problem. In the theory presented by Gale (1967) and McKenzie(1968, 1986), however, shadow prices supporting infinite-horizon paths is an integral aspect.

Unlike the golden-rule prices, the prices supporting a (non-stationary) maximal or optimal path are typically time varying. However, their role is similar. In any time period, at the prices associated with a maximal (or optimal) path, the activity chosen along the maximal (or optimal) path maximizes generalized profit [the utility plus the valuation of terminal subplots of land minus the valuation of initial plots of land, terminal and initial referring to the time period in question] among all possible activities in the transition possibility set. 
Roughly speaking, the analysis of forest management offered in Mitra and Wan (1986) follows Brock (1970a) quite closely. When it comes to studying long-run behavior of maximal paths, this theory again turns out to be the most elegant one to apply, as we have indicated in the previous section.

But, now, we need to concern ourselves with short-run behavior as well. If "errant" behavior can occur in the short-run along maximal paths, which would not occur on optimal paths (according to the overtaking criterion), this would destroy to some extent the importance of the concept of maximal paths. The fact that such errant behavior cannot occur in the short-run along maximal paths is at the heart of establishing an equivalence between a maximal path and an optimal path. In order to demonstrate this, we need to find shadow prices to support a maximal path. Thus, the theory relating to shadow prices, proposed by Gale (1967) and McKenzie $(1968,1986)$, becomes very useful in the present context.

Unfortunately, a part of this theory cannot be applied directly, because the model of forestry has characteristics which violate some of the standard assumptions under which the general theory of optimal intertemporal allocation has been developed. We use a version of the Kuhn-Tucker theorem due to Arrow, Hurwicz and Uzawa (1961), and then follow the Gale-McKenzie approach to obtain the appropriate shadow prices. The appropriate result is stated in Proposition 3; its proof is quite involved, although it uses methods familiar in the literature on the general theory of intertemporal allocation that we have repeatedly referred to.

To proceed with our analysis, we will make the following assumption ${ }^{25}$ on the utility function, $u$.

(A.4) $u$ is continuously differentiable on $\mathbb{R}_{+}$, with $B \equiv u^{\prime}(0)<\infty$.

We define a function $w: \mathbb{R} \rightarrow \mathbb{R}$ by:

$$
w(c)= \begin{cases}u(c) & \text { for } \quad c \in I \\ u^{\prime}(0) c & \text { for } c<0 \\ u^{\prime}(1)(c-1)+u(1) & \text { for } \quad c>1\end{cases}
$$

\footnotetext{
${ }^{25}$ While this assumption is crucial to our method of investigation (duality theory), it is not clear whether it is indispensable for the results of the next section on the relation between maximal and optimal paths. It would seem that a "primal route" to those results should be possible. See Mitra (2003) for such an approach in the context of aggregative models of economic growth and renewable resources. In any case, the duality theory developed in the current section (using (A.4)) might be of interest, independent of its application to obtain the results of the next section.
} 
Then $w$ is concave and continuously differentiable on $\mathbb{R}$, with $w(c)=u(c)$ for $c \in L$. Define $W: \mathbb{R}^{2 n} \rightarrow \mathbb{R}$ by:

$$
W(y, z)=w(P(y-A z))
$$

Then $W$ is continuously differentiable on its domain.

Proposition 3 Suppose $\left(\tilde{y}_{t}\right)_{1}^{\infty}$ is a maximal path from $\tilde{y} \in Q$. Then, there is $N \in \mathbb{N}, N>1$, and a sequence of shadow prices $\left(\tilde{p}_{t}\right)_{N-1}^{\infty}$ such that:

(i) $\tilde{p}_{t} \in \mathbb{R}_{+}^{n}$ and $\tilde{p}_{t} \leq(2 n+1) B b$ for all $t \in \mathbb{N}$, with $t \geq N-1$;

(ii) If $\left(y_{t}\right)_{1}^{\infty}$ is a path from $\tilde{y} \in Q$, then:

$$
\sum_{t=1}^{N-1} u\left(P\left(y_{t}-A y_{t+1}\right)\right)+\tilde{p}_{N} y_{N} \leq \sum_{t=1}^{N-1} u\left(P\left(\tilde{y}_{t}-A \tilde{y}_{t+1}\right)\right)+\tilde{p}_{N} \tilde{y}_{N}
$$

(iii) For $t \geq N-1$, and for all $(y, z) \in \Omega$,

$$
u(P(y-A z))+\tilde{p}_{t+1} z-\tilde{p}_{t} y \leq u\left(P\left(\tilde{y}_{t}-A \tilde{y}_{t+1}\right)\right)+\tilde{p}_{t+1} \tilde{y}_{t+1}-\tilde{p}_{t} \tilde{y}_{t}
$$

Proof. Since $\left(\tilde{y}_{t}\right)_{1}^{\infty}$ is maximal, given any $T \in \mathbb{N},\left(\tilde{y}_{1}, \ldots, \tilde{y}_{T+1}\right)$ must solve the following constrained maximization problem:

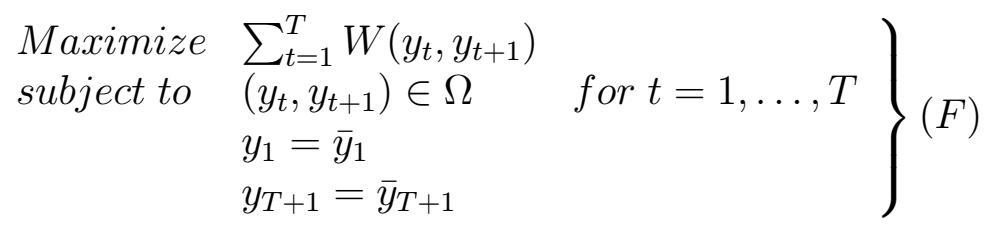

Suppose on the contrary that there is $\left(y_{1}, \ldots, y_{T+1}\right)$, satisfying the constraints of problem $(\mathrm{F})$, for which the following holds:

$$
W\left(y_{t}, y_{t+1}\right)>W\left(\tilde{y}_{t}, \tilde{y}_{t+1}\right)
$$

Then, we can define $\left(y_{t}^{\prime}\right)_{1}^{\infty}$ as: $y_{t}^{\prime}=y_{t}$ for $t=1, \ldots, T+1$, and $y_{t}^{\prime}=\tilde{y}_{t}$ for $t>T+1$. Then, it is easy to check that $\left(y_{t}^{\prime}\right)_{1}^{\infty}$ is a path from $y$, for which $c_{t}^{\prime}=\tilde{c}_{t}$ for $t \geq T+1$. Then, using (38), we would have:

$$
\sum_{t=1}^{T} u\left(P\left(y_{t}^{\prime}-A y_{t+1}^{\prime}\right)\right)>\sum_{t=1}^{T} u\left(P\left(\tilde{y}_{t}-A \tilde{y}_{t+1}\right)\right)
$$

a contradiction to the fact that $\left(\tilde{y}_{t}\right)_{1}^{\infty}$ is maximal. 
We now proceed to examine the constrained maximum problem. In order to apply the Kuhn-Tucker theorem to it, we write it first in the "standard form". Define $X=\mathbb{R}^{n}$, and $Y=X^{T+1}$. Then, $\left(\tilde{y}_{1}, \ldots, \tilde{y}_{T+1}\right)$ must solve the following problem:

$$
\left.\begin{array}{lll}
\begin{array}{l}
\text { Maximize } \\
\text { subject to }
\end{array} & \sum_{t=1}^{T} W\left(y_{t}, y_{t+1}\right) & \\
& y_{t}-A y_{t+1} \geq 0 \quad \text { for } t=1, \ldots, T \\
& y_{t} \geq 0 & \text { for } t=1, \ldots, T+1 \\
& y_{1}-\tilde{y}_{1} \geq 0 & \\
& \tilde{y}_{1}-y_{1} \geq 0 & \\
& y_{T+1}-\tilde{y}_{T+1} \geq 0 & \\
& \tilde{y}_{T+1}-y_{T+1} \geq 0 & \\
& 1-\sum_{i=1}^{n} y_{t}^{i} \geq 0 & \text { for } t=1, \ldots, T+1 \\
& \sum_{i=1}^{n} y_{t}^{i}-1 \geq 0 & \text { for } t=1, \ldots, T+1
\end{array}\right\}
$$

among all $\left(y_{1}, \ldots, y_{T+1}\right) \in Y$. Notice that the set $Y$ is open. The constraint functions are all linear, and hence continuously differentiable on $Y$. The objective function is continuously differentiable on $Y$, by the way $W$ was defined and assumption (A.4). The Arrow-Hurwicz-Uzawa constraint qualification is met since the constraint functions are all linear. Thus, we can apply their version of the Kuhn-Tucker theorem to obtain $\left(r_{1}, \ldots, r_{T}\right) \geq 0,\left(\nu_{1}, \ldots, \nu_{T+1}\right) \geq$ $0, \alpha \geq 0, \alpha^{\prime} \geq 0, \omega \geq 0, \omega^{\prime} \geq 0,\left(\mu_{1}, \ldots, \mu_{T+1}\right) \geq 0,\left(\mu_{1}^{\prime}, \ldots, \mu_{T+1}^{\prime}\right) \geq 0$, such that:

$$
\begin{array}{r}
-u^{\prime}\left(\tilde{c}_{t}\right) P A+u^{\prime}\left(\tilde{c}_{t+1}\right) P+\left[r_{t+1}-r_{t} A\right]+\nu_{t+1}-\mu_{t+1} b+\mu_{t+1}^{\prime} b= \\
\text { for } t=1, \ldots, T-1 \\
u^{\prime}\left(\tilde{c}_{1}\right) P+r_{1}+\nu_{1}+\alpha-\alpha^{\prime}-\mu_{1} b+\mu_{1}^{\prime} b=0 \\
-u^{\prime}\left(\tilde{c}_{T}\right) P A-r_{T} A+\nu_{T+1}+\omega-\omega^{\prime}-\mu_{T+1} b+\mu_{T+1}^{\prime} b=0 \\
r(t)\left(\tilde{y}_{t}-A \tilde{y}_{t+1}\right)=0 \text { for } t=1, \ldots, T-1 \\
\nu(t) \tilde{y}(t)=0 \text { for } t=1, \ldots, T+1 \\
\alpha\left(y_{1}-\tilde{y}_{1}\right)+\alpha^{\prime}\left(\tilde{y}_{1}-y_{1}\right)=0 \\
\omega\left(y_{T+1}-\tilde{y}_{T+1}\right)+\omega^{\prime}\left(\tilde{y}_{T+1}-y_{T+1}\right)=0 \\
\mu_{t}\left(1-\sum_{i=1}^{n} \tilde{y}_{t}^{i}\right)+\mu_{t}^{\prime}\left(\sum_{i=1}^{n} \tilde{y}_{t}^{i}-1\right)=0
\end{array}
$$


Let us define $\left(q_{1}, \ldots, q_{T}\right)$ by $q_{t}=u^{\prime}\left(\tilde{c}_{t}\right) P$ for $t=1, \ldots, T$, and $\left(p_{1}, \ldots, p_{T}\right)$ by $p_{t}=q_{t}+r_{t}$ for $t=1, \ldots, T$. Now, let us consider any $(y, z) \in \Omega$, and $t \in\{1, \ldots, T-1\}$. Then, using (39), we have:

$$
\begin{aligned}
q_{t} A z & =q_{t+1} z+\left[r_{t+1}-r_{t} A\right] z+\nu_{t+1} z-\mu_{t+1} b z+\mu_{t+1}^{\prime} b z \\
& =q_{t+1} z+\left[r_{t+1}-r_{t} A\right] z+\nu_{t+1} z-\mu_{t+1}+\mu_{t+1}^{\prime}
\end{aligned}
$$

Using (47), we obtain:

$$
q_{t} y-q_{t} A z=q_{t} y-q_{t+1} z-\left[r_{t+1}-r_{t} A\right] z-\nu_{t+1} z+\mu_{t+1}-\mu_{t+1}^{\prime}
$$

Using (A.4), we have:

$$
\begin{aligned}
u(P(y-A z))-u\left(P\left(\tilde{y}_{t}-A \tilde{y}_{t+1}\right)\right) & \leq u^{\prime}\left(\tilde{c}_{t}\right)\left[P(y-A z)-P\left(\tilde{y}_{t}-A \tilde{y}_{t+1}\right)\right] \\
& =q_{t} y-q_{t} A z-\left[q_{t} \tilde{y}_{t}-q_{t} A \tilde{y}_{t+1}\right]
\end{aligned}
$$

Using (42),(43) and (48) in (49), we obtain:

$$
\begin{aligned}
u(P(y-A z))-u\left(P\left(\tilde{y}_{t}-A \tilde{y}_{t+1}\right)\right) \leq & q_{t} y-q_{t+1} z-\left[r_{t+1}-r_{t} A\right] z-\nu_{t+1} z \\
& -\left[q_{t} \tilde{y}_{t}-q_{t+1} \tilde{y}_{t+1}-\left[r_{t+1}-r_{t} A\right] \tilde{y}_{t+1}\right] \\
\leq & q_{t} y-q_{t+1} z-r_{t+1} z+r_{t} y \\
& -\left[q_{t} \tilde{y}_{t}-q_{t+1} \tilde{y}_{t+1}-r_{t+1} \tilde{y}_{t+1}+r_{t} \tilde{y}_{t}\right] \\
= & p_{t} y-p_{t+1} z-\left[p_{t} \tilde{y}_{t}-p_{t+1} \tilde{y}_{t+1}\right]
\end{aligned}
$$

Transposing terms, we see that for all $(y, z) \in \Omega$, and $t \in\{1, \ldots, T-1\}$,

$$
u(P(y-A z))+p_{t+1} z-p_{t} y \leq u\left(P\left(\tilde{y}_{t}-A \tilde{y}_{t+1}\right)\right)+p_{t+1} \tilde{y}_{t+1}-p_{t} \tilde{y}_{t}
$$

To summarize this first part of the proof, given $T \in \mathbb{N}$, we have obtained $\left(p_{1}, \ldots, p_{T}\right) \geq 0$, such that for all $(y, z) \in \Omega$, and $t \in\{1, \ldots, T-1\},(50)$ holds.

The vector $\left(p_{1}, \ldots, p_{T}\right)$ obtained above depends, of course, on the $T \in \mathbb{N}$ that is given to begin with, and one would write $\left(p_{1}\{T\}, \ldots, p_{T}\{T\}\right)$ to record this explicitly . In order to obtain an infinite sequence $\left(\bar{p}_{t}\right)_{1}^{\infty}$ from these finite vectors, one would use a Cantor diagonal process argument; a pre-requisite to applying this argument is to show that for each $t \in \mathbb{N}$, there is a real number $B_{t}$, such that $\left\|p_{t}\{T\}\right\| \leq B_{t}$ for all $T \geq t$.

It turns out that showing that such bounds exist for all $t \in \mathbb{N}$ is somewhat problematic in the forestry model. Specifically, showing that there are such 
bounds on all $r_{t}\{T\}$ creates difficulties. However, it is possible to show that there is $N \in \mathbb{N}$, such that bounds exist for all $t \geq N$. This is why the statement of our theorem is split up into two parts, instead of the standard Gale-McKenzie formulation, where (38) would be established for all $t \in \mathbb{N}$.

We now proceed with the details of the second part of the proof. Since $\left(\tilde{y}_{t}\right)_{1}^{\infty}$ is maximal, it is good. So, we can find $N^{\prime} \in \mathbb{N}$, such that for all $t \geq N^{\prime}$,

$$
\tilde{y}_{t}^{i} \geq(1 / 2 m) \text { for } i=1, \ldots, m ; \tilde{h}_{t}^{m} \geq(1 / 2 m)
$$

We confine our attention henceforth to those $T \in \mathbb{N}$ which satisfy $T>N^{\prime}+$ $n$. Given any such $T$, we have non-negative vectors $\left(r_{1}\{T\}, \ldots, r_{T}\{T\}\right),\left(q_{1}\{T\}, \ldots, q_{T}\{T\}\right)$ and $\left(p_{1}\{T\}, \ldots, p_{T}\{T\}\right)$ as defined above, satisfying for all $(y, z) \in \Omega$, and $t \in\{1, \ldots, T-1\}$,

$u(P(y-A z))+p_{t+1}\{T\} z-p_{t}\{T\} y \leq u\left(P\left(\tilde{y}_{t}-A \tilde{y}_{t+1}\right)\right)+p_{t+1}\{T\} \tilde{y}_{t+1}-p_{t}\{T\} \tilde{y}_{t}$

Clearly, using the above definition of $q_{t}\{T\}$ and (A.4), we have:

$$
q_{t}\{T\} \leq B P \leq B b \text { for } t \in\{1, \ldots, T\}
$$

It remains to find appropriate bounds for $r_{t}\{T\}$.

Define $\eta_{t+1}\{T\}=\mu_{t+1}\{T\}-\mu_{t+1}^{\prime}\{T\}$ for $t=1, \ldots, T+1$. Using (39), we get:

$$
\begin{aligned}
\eta_{t+1}\{T\} b & =-q_{t}\{T\} A+q_{t+1}\{T\}-r_{t}\{T\} A+r_{t+1}\{T\}+\nu_{t+1}\{T\} \\
& \geq-q_{t}\{T\} A-r_{t}\{T\} A
\end{aligned}
$$

Since the first component of the vector on the right hand side of (53) is 0 , and $b=(1, \ldots, 1)$, we must have $\eta_{t+1}\{T\} \geq 0$.

Using (39) again and noting that:

$$
\begin{aligned}
\eta_{t+1}\{T\} b & =-q_{t}\{T\} A+q_{t+1}\{T\}-r_{t}\{T\} A+r_{t+1}\{T\}+\nu_{t+1}\{T\} \\
& \leq q_{t+1}\{T\}+r_{t+1}\{T\}+\nu_{t+1}\{T\}
\end{aligned}
$$

we have:

$$
\eta_{t+1}\{T\} \leq q_{t+1}^{m}\{T\}+r_{t+1}^{m}\{T\}+\nu_{t+1}^{m}\{T\}
$$

For $t \geq N^{\prime}$, we have $\tilde{h}_{t}^{m} \geq(1 / 2 m)$, and $\tilde{y}_{t}^{m} \geq(1 / 2 m)$. So by $(42)$, we have $r_{t}^{m}\{T\}=0$, and by (43), we have $\nu_{t}^{m}\{T\}=0$. Using this information in (54), together with (52) yields:

$$
\eta_{t+1}\{T\} \leq B \text { for } T \geq t \geq N^{\prime}-1
$$


Using (52) and (55) in (39), we have for $T \geq t \geq N^{\prime}-1$,

$$
\begin{aligned}
r_{t+1}\{T\}-r_{t}\{T\} A & =q_{t}\{T\} A-q_{t+1}\{T\}-\nu_{t+1}\{T\}+\eta_{t+1}\{T\} b \\
& \leq q_{t}\{T\} A+\eta_{t+1}\{T\} b \\
& \leq 2 B b
\end{aligned}
$$

We can now use (56) to obtain:

$$
\left.\begin{array}{lll}
r_{t+1}^{1}\{T\} \leq 2 B & \text { for } & t \geq N^{\prime}-1 \\
r_{t+1}^{2}\{T\} \leq 4 B & \text { for } & t \geq N^{\prime} \\
r_{t+1}^{3}\{T\} \leq 6 B & \text { for } & t \geq N^{\prime}+1 \\
\cdots & \cdots & \cdots \\
r_{t+1}^{n}\{T\} \leq 2 n B & \text { for } & t \geq N^{\prime}+n-2
\end{array}\right\}
$$

This implies that:

$$
r_{t+1}\{T\} \leq 2 n B b \text { for } T \geq t \geq N^{\prime}+n-2
$$

Thus, denoting $\left(N^{\prime}+n\right)$ by $N$, we have:

$$
p_{t+1}\{T\} \leq(2 n+1) B b \text { for } T \geq t \geq N-2
$$

These are the bounds on the shadow prices that we need. This completes the second part of the proof.

Let $\left(y_{t}\right)_{1}^{\infty}$ be a path from $y \in Q$. Using (51), we obtain:

$$
\sum_{t=1}^{N-1}\left[u\left(P\left(y_{t}-A y_{t+1}\right)\right)-u\left(P\left(\tilde{y}_{t}-A \tilde{y}_{t+1}\right)\right) \leq p_{N}\{T\}\left(\tilde{y}_{N}-y_{N}\right)\right.
$$

We have now established that for each $T>N$, there is a vector $\left(p_{N-1}\{T\}, \ldots, p_{T}\{T\}\right)$, such that:

$$
0 \leq p_{t+1}\{T\} \leq(2 n+1) B b \text { for } N-2 \leq t \leq T-1
$$

holds, (58) holds, and for all $(y, z) \in \Omega$, and for all $N-1 \leq t \leq T-1$, $u(P(y-A z))+p_{t+1}\{T\} z-p_{t}\{T\} y \leq u\left(P\left(\tilde{y}_{t}-A \tilde{y}_{t+1}\right)\right)+p_{t+1}\{T\} \tilde{y}_{t+1}-p_{t}\{T\} \tilde{y}_{t}$ holds. 
Given (59), we can now use the Cantor diagonal process to obtain a subsequence $T^{\prime}$ of $T(>N)$, and a sequence $\left(\tilde{p}_{t}\right)_{N-1}^{\infty}$ such that, for each $t \geq$ $N-1$

$$
p_{t}\left(T^{\prime}\right) \rightarrow \tilde{p}_{t} \text { as } T^{\prime} \rightarrow \infty
$$

Using (61) in (58) yields (36). Using (61) in (60) yields (37). Using (61) in (59) yields:

$$
0 \leq \tilde{p}_{t} \leq(2 n+1) B b \text { for } t \geq N-1
$$

This completes the third and last part of the proof.

\section{Maximal Paths are Optimal}

In this section, we use the results of the previous two sections to show that the notions of maximality and optimality coincide for our forestry model.

To this end, recall from the definitions of optimal and maximal paths that an optimal path must be maximal. It is the reverse implication that is non-trivial and of significant interest.

To make the discussion non-void, we show (in Theorem 6) that there exists an optimal path from every initial forest, by following the method of Brock (1970a). Then, using the price support property of maximal paths (established in Proposition 3), we show (in Theorem 7) that a maximal path is necessarily optimal.

We first present our result on the existence of an optimal path. We provide a fairly self-contained treatment of this familiar topic in intertemporal allocation theory, because there are several differences between our framework and the one typically used in the standard version of this theory.

Theorem 6 There exists an optimal path $\left(\tilde{y}_{t}\right)_{1}^{\infty}$ from every $\tilde{y} \in Q$.

Proof. We first note that there is a good path from $\tilde{y}$. Since $(\tilde{y}, \bar{y}) \in Q^{2}$ [where $\bar{y}$ is the golden-rule] we can use Lemma 1 to obtain $\left(z_{1}, \ldots, z_{n+2}\right)$ such that:

$$
(i)\left(z_{s}, z_{s+1}\right) \in \Omega \text { for } s=1, \ldots, n+1 ;(i i) z_{1}=\tilde{y}, z_{n+2}=\bar{y} \text {. }
$$

Define a sequence $\left(y_{t}^{\prime}\right)_{1}^{\infty}$ as follows:

$$
\left.\begin{array}{l}
y_{t}^{\prime}=z_{t} \text { for } t=1, \ldots, n+2 \\
y_{t}^{\prime}=\bar{y} \text { for } t>n+2
\end{array}\right\}
$$


It is easy to check that $\left(y_{t}^{\prime}\right)_{1}^{\infty}$ is a path from $y \in Q$. Since $y_{t}^{\prime}=\bar{y}$ for $t>n+2$, we have $c_{t}^{\prime}=\bar{c}$ for $t>n+2$. It follows that for all $T>n+2$,

$$
\sum_{t=1}^{T}\left[u\left(P\left(y_{t}^{\prime}-A y_{t+1}^{\prime}\right)\right)-u(\bar{c})\right] \geq-2(n+1) u(\bar{c})
$$

Thus, $\left(y_{t}^{\prime}\right)_{1}^{\infty}$ is good.

Next, we claim that for any good path $\left(y_{t}\right)_{1}^{\infty}$ from $\tilde{y}$, we must have:

$$
\sum_{t=1}^{\infty} \delta_{t}<\infty
$$

To see this, observe that for any $T \in \mathbb{N}$, we can use (6) to write:

$$
\begin{aligned}
\sum_{t=1}^{T}\left[u\left(P\left(y_{t}-A y_{t+1}\right)\right)-u(\bar{c})\right] & =\sum_{t=1}^{T}\left[p y_{t}-p y_{t+1}\right]-\sum_{t=1}^{T} \delta_{t} \\
& =\left[p y-p y_{T+1}\right]-\sum_{t=1}^{T} \delta_{t}
\end{aligned}
$$

Since $\left(y_{t}\right)_{1}^{\infty}$ is good, there is $G \in \mathbb{R}$ such that:

$$
\sum_{t=1}^{T}\left[u\left(P\left(y_{t}-A y_{t+1}\right)\right)-u(\bar{c})\right] \geq G \text { for all } T \in \mathbb{N}
$$

Thus, we obtain:

$$
\sum_{t=1}^{T} \delta_{t} \leq p y-G \leq n-G \text { for all } T \in \mathbb{N}
$$

Since $\delta_{t} \geq 0$ by (6), this establishes our claim.

We now define:

$$
\delta(\tilde{y})=\inf \left\{\sum_{t=0}^{\infty} \delta_{t}:\left(y_{t}\right)_{1}^{\infty} \text { is a good path from } x\right\}
$$

Since there is a good path from $x, \delta(\tilde{y})$ is well-defined and $\delta(\tilde{y})<\infty$. 
Given the definition of $\delta(\tilde{y})$, we can choose a sequence of paths $\left(y_{t}\{N\}\right)_{1}^{\infty}$ from $\tilde{y}$ (for $N=1,2,3, \ldots$ ) such that:

$$
\sum_{t=0}^{\infty} \delta_{t}\{N\} \leq \delta(\tilde{y})+(1 / N) \text { for } N=1,2,3, \ldots
$$

Since for each $N=1,2,3, \ldots$, we have $y_{t}\{N\} \in Q$ for $t \geq 1$, we can use the Cantor diagonal process to find a subsequence $N^{\prime}$ of $N$, and a sequence $\left(\tilde{y}_{t}\right)_{1}^{\infty}$ such that, for each $t \in \mathbb{N}$, we have

$$
y_{t}\left\{N^{\prime}\right\} \rightarrow \tilde{y}_{t} \text { as } N^{\prime} \rightarrow \infty
$$

It can be checked that $\left(\tilde{y}_{t}\right)_{1}^{\infty}$ is a path from $\tilde{y}$, and for each $t \in \mathbb{N}$, we have:

$$
\delta_{t}\left\{N^{\prime}\right\} \rightarrow \tilde{\delta}_{t} \text { as } N^{\prime} \rightarrow \infty
$$

We claim that the sequence $\left(\tilde{\delta}_{t}\right)_{1}^{\infty}$ satisfies:

$$
\delta(\tilde{y})=\sum_{t=1}^{\infty} \tilde{\delta}_{t}
$$

If the claim is not true, then we can find $T \in \mathbb{N}$, such that:

$$
\delta(\tilde{y})<\sum_{t=1}^{T} \tilde{\delta}_{t}
$$

Defining $\gamma=\sum_{t=1}^{T} \tilde{\delta}_{t}$, pick a number $\gamma^{\prime}$ such that $\gamma>\gamma^{\prime}>\delta(\tilde{y})$. Then, we can find $\tilde{N}$, such that for all $N^{\prime} \geq \tilde{N}$,

$$
\gamma^{\prime}<\sum_{t=1}^{T} \delta_{t}\left\{N^{\prime}\right\}
$$

Thus, for all $N^{\prime} \geq \tilde{N}$, we have:

$$
\gamma^{\prime}<\sum_{t=1}^{T} \delta_{t}\left\{N^{\prime}\right\} \leq \sum_{t=1}^{\infty} \delta_{t}\left\{N^{\prime}\right\} \leq \delta(\tilde{y})+\left(1 / N^{\prime}\right)
$$

Letting $N^{\prime} \rightarrow \infty$, we get $\gamma^{\prime} \leq \delta(\tilde{y})$, which contradicts the definition of $\gamma^{\prime}$. This establishes our claim. 
Consider the path $\left(\tilde{y}_{t}\right)_{1}^{\infty}$ from $\tilde{y}$, defined above. It is easy to check that $\left(\tilde{y}_{t}\right)_{1}^{\infty}$ is a good path. We will show that it is an optimal path from $\tilde{y}$. Suppose, on the contrary, there is a path $\left(y_{t}^{\prime}\right)_{1}^{\infty}$ from $\tilde{y}$, such that the associated consumption sequences $\left(c_{t}^{\prime}\right)_{1}^{\infty}$ and $\left(\tilde{c}_{t}\right)_{1}^{\infty}$ satisfy the condition that $\left(c_{t}^{\prime}\right)_{1}^{\infty} R_{u}\left(\tilde{c}_{t}\right)_{1}^{\infty}$ [where the pre-order $R_{u}$ is defined by $(\mathrm{R})$ ]. This will be the case if and only if there is $\bar{N} \in \mathbb{N}$, such that:

$$
\sum_{t=1}^{N} u\left(c_{t}^{\prime}\right) \geq \sum_{t=1}^{N} u\left(\tilde{c}_{t}\right) \text { for all } N \geq \bar{N}
$$

and :

$$
\sum_{t=1}^{N^{\prime}} u\left(c_{t}^{\prime}\right)>\sum_{t=1}^{N^{\prime}} u\left(\tilde{c}_{t}\right) \text { for a subsequence } N^{\prime} \text { of } N \geq \bar{N}
$$

Define a sequence $\left(y_{t}^{\prime \prime}\right)_{1}^{\infty}$ by: $y_{t}^{\prime \prime}=\left(\frac{1}{2}\right) y_{t}^{\prime}+\left(\frac{1}{2}\right) \tilde{y}_{t}$ for $t \in \mathbb{N}$. It is easy to check that $\left(y_{t}^{\prime \prime}\right)_{1}^{\infty}$ is a path from $\tilde{y}$, and its associated consumption sequence $\left(c_{t}^{\prime \prime}\right)_{1}^{\infty}$ satisfies: $c_{t}^{\prime \prime}=\left(\frac{1}{2}\right) c_{t}^{\prime}+\left(\frac{1}{2}\right) \tilde{c}_{t}$ for $t \in \mathbb{N}$. Using (64), we know that $c_{t}^{\prime} \neq \tilde{c}_{t}$ for some $t=\tau \in \mathbb{N}$. Then, by concavity of $u$, and strict mid-concavity of $u$, we have:

$$
\begin{aligned}
& \text { (a) } u\left(c_{t}^{\prime \prime}\right) \geq\left(\frac{1}{2}\right) u\left(c_{t}^{\prime}\right)+\left(\frac{1}{2}\right) u\left(\tilde{c}_{t}\right) \text { for all } t \in \mathbb{N} \\
& \text { (b) } \xi \equiv u\left(c_{t}^{\prime \prime}\right)-\left[\left(\frac{1}{2}\right) u\left(c_{t}^{\prime}\right)+\left(\frac{1}{2}\right) u\left(\tilde{c}_{t}\right)\right]>0 \text { for } t=\tau
\end{aligned}
$$

Defining $\tilde{N}=\max [\bar{N}, \tau]$, and using (65) in (63), we get:

$$
\xi \leq\left[\sum_{t=1}^{N} u\left(c_{t}^{\prime \prime}\right)-\sum_{t=1}^{N} u\left(\tilde{c}_{t}\right)\right] \text { for all } N \geq \tilde{N}
$$

This shows that $\left(y_{t}^{\prime \prime}\right)_{1}^{\infty}$ is also a good path from $\tilde{y}$.

We can now use (6) to write for all $N \in \mathbb{N}$,

$$
\begin{aligned}
{\left[\sum_{t=1}^{N} u\left(c_{t}^{\prime \prime}\right)-\sum_{t=1}^{N} u\left(\tilde{c}_{t}\right)\right] } & =\sum_{t=1}^{N}\left[u\left(P\left(y_{t}^{\prime \prime}-A y_{t+1}^{\prime \prime}\right)\right)-u\left(P\left(\tilde{y}_{t}-A \tilde{y}_{t+1}\right)\right)\right] \\
& =\left[p \tilde{y}_{N+1}-p y_{N+1}^{\prime \prime}\right]-\sum_{t=1}^{N} \delta_{t}^{\prime \prime}+\sum_{t=1}^{N} \tilde{\delta}_{t}
\end{aligned}
$$

Since $\left(y_{t}^{\prime \prime}\right)_{1}^{\infty}$ and $\left(\tilde{y}_{t}\right)_{1}^{\infty}$ are both good paths from $\tilde{y}$, we have $y_{N+1}^{\prime \prime} \rightarrow \bar{y}$ and $\tilde{y}_{N+1} \rightarrow \bar{y}$ as $N \rightarrow \infty$. Thus, we can choose $N_{1}$ such that for $N \geq N_{1}$, we have 
$\left[p \tilde{y}_{N+1}-p y_{N+1}^{\prime \prime}\right]<\left(\frac{1}{2}\right) \xi$. Given the definition of $\delta(\tilde{y})$ and the path $\left(\tilde{y}_{t}\right)_{1}^{\infty}$, we can choose $N_{2}$ such that for $N \geq N_{2}$, we have $\left[\sum_{t=1}^{N} \tilde{\delta}_{t}-\sum_{t=1}^{N} \delta_{t}^{\prime \prime}\right]<\left(\frac{1}{2}\right) \xi$. Thus, for $N>\max \left[N_{1}, N_{2}, \tilde{N}\right]$, we have from (67) that:

$$
\left[\sum_{t=1}^{N} u\left(c_{t}^{\prime \prime}\right)-\sum_{t=1}^{N} u\left(\tilde{c}_{t}\right)\right]<\xi
$$

But this contradicts (66) and establishes that $\left(\tilde{y}_{t}\right)_{1}^{\infty}$ is optimal from $\tilde{y}$.

Next, we show that a maximal path is necessarily optimal. This depends on the methods used by Gale (1967) and McKenzie (1968), and relies on the shadow prices associated with maximal paths, derived in the previous section.

Theorem 7 A path $\left(\tilde{y}_{t}\right)_{1}^{\infty}$ from $\tilde{y} \in Q$ is maximal if and only if it is optimal.

Proof. If a path $\left(\tilde{y}_{t}\right)_{1}^{\infty}$ from $\tilde{y} \in Q$ is optimal, then by definitions of optimality and maximality, $\left(\tilde{y}_{t}\right)_{1}^{\infty}$ is a maximal path from $\tilde{y} \in Q$.

To establish the converse result, let $\left(\tilde{y}_{t}\right)_{1}^{\infty}$ be a maximal path from $\tilde{y} \in Q$. We will show that it is optimal. Suppose this is not the case. Then there is a path $\left(y_{t}^{\prime}\right)_{1}^{\infty}$ from $\tilde{y}$, such that the associated consumption sequences $\left(c_{t}^{\prime}\right)_{1}^{\infty}$ and $\left(\tilde{c}_{t}\right)_{1}^{\infty}$ satisfy the condition that $\left(c_{t}^{\prime}\right)_{1}^{\infty} P_{u}\left(\tilde{c}_{t}\right)_{1}^{\infty}$ [where the pre-order $R_{u}$ is defined by (R)]. This will be the case if and only if there is $\bar{N} \in \mathbb{N}$, such that:

$$
\sum_{t=1}^{T} u\left(c_{t}^{\prime}\right) \geq \sum_{t=1}^{T} u\left(\tilde{c}_{t}\right) \text { for all } T \geq \bar{N}
$$

and :

$$
\sum_{t=1}^{T^{\prime}} u\left(c_{t}^{\prime}\right)>\sum_{t=1}^{T^{\prime}} u\left(\tilde{c}_{t}\right) \text { for a subsequence } T^{\prime} \text { of } T \geq \bar{N}
$$

Define a sequence $\left(y_{t}\right)_{1}^{\infty}$ by: $y_{t}=\left(\frac{1}{2}\right) y_{t}^{\prime}+\left(\frac{1}{2}\right) \tilde{y}_{t}$ for $t \in \mathbb{N}$. It is easy to check that $\left(y_{t}\right)_{1}^{\infty}$ is a path from $\tilde{y}$, and its associated consumption sequence $\left(c_{t}\right)_{1}^{\infty}$ satisfies: $c_{t}=\left(\frac{1}{2}\right) c_{t}^{\prime}+\left(\frac{1}{2}\right) \tilde{c}_{t}$ for $t \in \mathbb{N}$. Using (69), we know that $c_{t}^{\prime} \neq \tilde{c}_{t}$ for some $t=\tau \in \mathbb{N}$. Then, by concavity of $u$, and strict mid-concavity of $u$, we have:

$$
\begin{aligned}
& \text { (a) } u\left(c_{t}\right) \geq\left(\frac{1}{2}\right) u\left(c_{t}^{\prime}\right)+\left(\frac{1}{2}\right) u\left(\tilde{c}_{t}\right) \text { for all } t \in \mathbb{N} \\
& \text { (b) } \xi \equiv u\left(c_{t}\right)-\left[\left(\frac{1}{2}\right) u\left(c_{t}^{\prime}\right)+\left(\frac{1}{2}\right) u\left(\tilde{c}_{t}\right)\right]>0 \text { for } t=\tau
\end{aligned}
$$


Defining $\tilde{N}=\max [\bar{N}, \tau]$, and using (70) in (68), we get:

$$
\xi \leq\left[\sum_{t=1}^{T} u\left(c_{t}\right)-\sum_{t=1}^{T} u\left(\tilde{c}_{t}\right)\right] \text { for all } T \geq \tilde{N}
$$

Since $\left(\tilde{y}_{t}\right)_{1}^{\infty}$ is a maximal path from $\tilde{y} \in Q$, it is a good path from $\tilde{y}$, by Proposition 2. The inequality (71) shows that $\left(y_{t}\right)_{1}^{\infty}$ is also a good path from $\tilde{y}$.

Since $\left(\tilde{y}_{t}\right)_{1}^{\infty}$ is a maximal path from $\tilde{y} \in Q$, we can use Proposition 3 to obtain $N \in \mathbb{N}, N>1$, and a sequence of shadow prices $\left(\tilde{p}_{t}\right)_{N-1}^{\infty}$ such that:

$$
0 \leq \tilde{p}_{t} \leq(2 n+1) B b \text { for all } t \in \mathbb{N} \text {, with } t \geq N-1
$$

and (36) and (37) hold. Defining $\hat{N}=\max [N, \tilde{N}]$, and using (36) and (37), we have for $T>\hat{N}$,

$$
\begin{aligned}
{\left[\sum_{t=1}^{T} u\left(c_{t}\right)-\sum_{t=1}^{T} u\left(\tilde{c}_{t}\right)\right] } & =\sum_{t=1}^{T}\left[u\left(P\left(y_{t}-A y_{t+1}\right)\right)-u\left(P\left(\tilde{y}_{t}-A \tilde{y}_{t+1}\right)\right)\right] \\
& \leq \tilde{p}_{T+1}\left(\tilde{x}_{T+1}-x_{T+1}\right)
\end{aligned}
$$

Since $\left(y_{t}\right)_{1}^{\infty}$ and $\left(\tilde{y}_{t}\right)_{1}^{\infty}$ are both good paths from $\tilde{y}$, we have $y_{T+1} \rightarrow \bar{y}$ and $\tilde{y}_{T+1} \rightarrow \bar{y}$ as $T \rightarrow \infty$. Thus, using (72), we must have $\tilde{p}_{T+1}\left(\tilde{x}_{T+1}-x_{T+1}\right) \rightarrow 0$ as $T \rightarrow \infty$. Using this in (73) contradicts (71) and establishes the result.

\section{Concluding Remarks}

Our analysis of forest management has tried to provide a more satisfactory basis for focusing on maximum sustained yield of timber as a key concept, given the objective of intergenerational equity. Since forest management involves much more than timber production, it would be interesting to generalize the analysis to a setting of sustainable forest management, where all forest products are taken into account. Since, in principle, such an exercise would still fit into the general theory of intertemporal allocation, we feel that such a generalization would be theoretically feasible. The inter-relationships that would have to prevail between the various forest products at the maximum sustained forest yield solution would reflect the actual biological features governing the (joint production) of forest products, and should be of interest to foresters. 
We note that, even as a model of timber management, our framework is greatly oversimplified, and we comment on possible generalizations. (i) Harvesting and replanting costs can be incorporated into the model fairly easily. (ii) All trees coming to maturity at the same time might have a lower timber yield per tree compared to a situation in which trees come to maturity on different subplots in different years. This aspect is less straightforward to incorporate into our model, because of the nature of the biological interaction among trees that is being captured. In such a scenario, one would expect to see (at least in transition) the phenomenon of "forest thinning" on maximal paths. We feel, however, that such considerations would tend to reinforce the concept of the golden-rule forest in the long-run, since the adverse effect being captured would be fairly minimal for the golden-rule solution. (iii) The assumption of strong convexity (Axiom 6) has been used extensively in our analysis. Without it, the uniqueness of a maximal path is unlikely to obtain. But, then, the uniqueness of an optimal path will not hold in general either. ${ }^{26}$ Whether maximal paths nevertheless continue to be optimal in the more general context, where strong convexity is replaced by (weak) convexity, remains an open question.

Our setting for forest management is best applied to governments managing forest resources of a country. Roughly, two-thirds of the world's forests are currently managed by governments, so we feel the model has fairly wide potential use. Of course, our analysis focuses on the managed forest, under intergenerational equity. Thus, the social rate of discount is zero, while the market rate of interest is typically positive. In the same vein, the shadow prices supporting maximal paths provide the correct social evaluation of forestry land, according to the vintage of trees standing on it, and are likely to differ from market prices of forestry land. This disparity arises because market prices and interest rates are determined by (aggregate) decisions of individuals whose preferences reflect fairly short-run objectives. In the interest of intergenerational equity (of generations yet unborn), governments can play a vital role in managing forests on a sustainable basis.

Our theory of shadow prices supporting maximal paths shows that judicious forest management involves the methods of social project evaluation, where the notion of "generalized profit maximization" is still central (as in private project evaluation), but the profits are evaluated at the appropri-

\footnotetext{
${ }^{26}$ In the context of a somewhat different dynamic optimization model, this has been demonstrated in a concrete example by Khan and Mitra (2002).
} 
ate shadow prices, and using the appropriate social rate of discount, rather than the corresponding market based magnitudes. In this respect, the considerations which lead a country to preserve its national parks are also the considerations which lead to forest management which respects intergenerational equity. 


\section{References}

[1] Arrow, K.J., L.Hurwicz and H.Uzawa: Constraint Qualifications in Maximization Problems, Naval Research Logistics Quarterly, 8 (1961).

[2] Asheim, G.B. and W. Buchholz, Can Stock-Specific Constraints be Justified ?, Paper presented at the ICESFM conference, University of Toronto, May 2004.

[3] Asheim, G.B. and B. Tungodden, Resolving Distributional Conflicts Between Generations, Economic Theory, 24 (2004), 221-230.

[4] Atsumi, H.: Neoclassical Growth and the Efficient Program of Capital Accumulation, Review of Economic Studies, 32 (1965), 127-136.

[5] Basu, K. and T. Mitra: Aggregating Infinite Utility Streams with Intergenerational Equity: The Impossibility of Being Paretian, Econometrica 71 (2003a), 1557-1563.

[6] Basu, K. and T.Mitra: Utilitarianism for Infinite Utility Streams: A New Welfare Criterion and its Axiomatic Characterization," CAE Working Paper 03-05, Cornell University, (2003b).

[7] Brock, W. A.: On Existence of Weakly Maximal Programmes in a MultiSector Economy, Review of Economic Studies, 37 (1970a), 275-280.

[8] Brock, W.A.: An Axiomatic Basis for the Ramsey-Weizsacker Overtaking Criterion, Econometrica 38 (1970b), 927-929.

[9] Debreu, G.: Representation of a Preference Ordering by a Numerical Function, Chapter 11 in R.M. Thrall, C.H. Coombs, R.L. Davis, Eds., Decision Processes; New York, John Wiley, 1954, 159-165.

[10] Debreu, G.: Theory of Value, New York: John Wiley, 1959.

[11] Debreu, G.: Topological Methods in Cardinal Utility Theory, in K. J. Arrow, S. Karlin and P. Suppes, Eds., Mathematical Methods in the Social Sciences; Stanford, Stanford University Press, 1960.

[12] Debreu, G. and T.C. Koopmans: Additively Decomposed Quasiconvex Functions, Mathematical Programming, 24 (1982), 1-38. 
[13] Gale, D.: On Optimal Development in a Multi-Sector Economy, Review of Economic Studies, 34 (1967),1-18.

[14] Gale, D.: A Geometrical Duality Theorem with Economic Applications, Review of Economic Studies, 34 (1967), 19-24.

[15] Khan, M.A.: Intertemporal Ethics, Modern Capital Theory and the Economics of Forestry, Paper presented at the ICESFM conference, University of Toronto, May 2004.

[16] Khan, M.A. and T. Mitra : Optimal Growth in the Robinson-SolowSrinivasan Model: the Two-Sector Setting Without Discounting, mimeo, Cornell University (2002).

[17] Koopmans, T.C.: Stationary Ordinal Utility and Impatience, Econometrica 28 (1960), 287-309.

[18] Koopmans, T.C.: Representation of Preference Orderings Over Time, Chapter 4 in C.B. McGuire and R. Radner, Eds., Decision and Organization, New York: North-Holland, 1972, 79-100.

[19] Koopmans, T.C., P.A. Diamond, and R.E. Williamson: Stationary Utility and Time Perspective, Econometrica 32 (1964), 82-100.

[20] Leontief, W.: A Note on the Interrelation of Subsets of Independent Variables of a Continuous Function with Continuous First Derivatives, Bulletin of the American Mathematical Society, 53 (1947), 343-350.

[21] Leontief, W.: Introduction to a Theory of the Internal Structure of Functional Relationships, Econometrica, 15 (1947), 361-373.

[22] Malinvaud, E.: Capital Accumulation and Efficient Allocation of Resources, Econometrica 21 (1953), 233-268.

[23] McKenzie, L. W.: Accumulation Programs of Maximum Utility and the von Neumann Facet, in J. N. Wolfe (ed.) Value, Capital and Growth, Edinburgh: Edinburgh University Press, 1968, 353-383.

[24] McKenzie, L. W.: Optimal Economic Growth, Turnpike Theorems and Comparative Dynamics, in K. J. Arrow and M. Intrilligator (eds.) Handbook of Mathematical Economics, Vol. 3, New York: North-Holland Publishing Company, 1986, 1281-1355. 
[25] Mitra, T.: Representation of Equitable Preferences with Applications to Aggregative Models of Economic Growth and Renewable Resources, mimeo, 2003.

[26] Mitra, T. and H.Y. Wan, Jr.: On the Faustmann Solution to the Forest Management Problem, Journal of Economic Theory, 40 (1986), 229-249.

[27] Radner, R.: Paths of Economic Growth that are Optimal with Regard Only to Final States: A Turnpike Theorem, Review of Economic Studies, 28 (1961), 98-104.

[28] Ramsey, F.: A Mathematical Theory of Savings, Economic Journal, 38 (1928), 543-559.

[29] Samuelson, P.A.: Foundations of Economic Analysis, Cambridge,Mass.:Harvard University Press, 1947.

[30] Samuelson, P.A.: Economics of Forestry in an Evolving Society, Economic Enquiry 14 (1976), 466-492.

[31] Sen, A.K.: Collective Choice and Social Welfare; Edinburgh, Oliver\&Boyd, 1971.

[32] Suppes, P.: Some Formal Models of Grading Principles, Synthese 6 (1966), 284-306.

[33] Svensson, L.-G.: Equity among Generations, Econometrica 48 (1980), 1251-1256.

[34] von Weizsäcker, C. C.: Existence of Optimal Programs of Accumulation for an Infinite Time Horizon, Review of Economic Studies, 32 (1965), 85-104.

[35] Yaari, M.E.: A Note on Separability and Quasiconcavity, Econometrica, 45 (1977), 1183-1186. 